\title{
Multi-wavelength study of the gravitational lens system RXS J1131-1231
}

\section{Long slit spectroscopy: micro-lensing probes the QSO structure ${ }^{\star}$}

\author{
D. Sluse ${ }^{1,2}$, J.-F. Claeskens ${ }^{2}$, D. Hutsemékers ${ }^{2, \star \star}$, and J. Surdej ${ }^{2, \star \star \star}$ \\ ${ }^{1}$ Laboratoire d'Astrophysique, École Polytechnique Fédérale de Lausanne (EPFL) Observatoire, 1290 Sauverny, Switzerland \\ e-mail: dominique.sluse@epfl.ch \\ ${ }^{2}$ Institut d'Astrophysique et de Géophysique, Université de Liège, Allée du 6 Août 17, B5C, 4000 Sart Tilman, Belgium
}

Received 27 November 2006 / Accepted 26 February 2007

\section{ABSTRACT}

\begin{abstract}
Aims. We discuss and characterize micro-lensing among the 3 brightest lensed images (A-B-C) of the gravitational lens system RXS J1131-1231 (a quadruply imaged AGN) by means of long slit optical and NIR spectroscopy. Qualitative constraints on the size of different emission regions are derived. We also perform a spectroscopic study of two field galaxies located within 1.6 arcmin radius from the lens.

Methods. We decompose the spectra into their individual emission components using a multi-component fitting approach. A complementary decomposition of the spectra enables us to isolate the macro-lensed fraction of the spectra independently of any spectral modelling.

Results. 1. The data support micro-lensing de-amplification of images A and C. Not only is the continuum emission microlensed in those images but also a fraction of the Broad Line emitting Region (BLR).

2. Micro-lensing of a very broad component of $\mathrm{Mg}$ II emission line suggests that the corresponding emission occurs in a region more compact than the other components of the emission line.

3. We find evidence that a large fraction of the Fe II emission arises in the outer parts of the BLR. We also find a very compact emitting region in the ranges 3080-3540 $\AA$ and 4630-4800 $\AA$ that is likely associated with Fe II.

4. The [O III] narrow emission line regions are partly spatially resolved. This enables us to put a lower limit of $\sim 110 h^{-1} \mathrm{pc}$ on their intrinsic size.

5. Analysis of $\mathrm{Mg}$ II absorption found in the spectra indicates that the absorbing medium is intrinsic to the quasar, has a covering factor of $20 \%$, and is constituted of small clouds homogeneously distributed in front of the continuum and BLRs.

6. Two neighbour galaxies are detected at redshifts $z=0.10$ and $z=0.289$. These galaxies are possible members of galaxy groups reported at those redshifts.
\end{abstract}

Key words. gravitational lensing - galaxies: Seyfert

\section{Introduction}

RXS J113155.4-123155 (hereafter J1131) is one of the nearest confirmed multiply imaged AGN. The source at $z_{\mathrm{s}}=0.66$ is lensed by an elliptical galaxy at $z_{1}=0.295$ (Sluse et al. 2003). This system is a long axis quad with an image configuration very similar to B1422+231 (Patnaik et al. 1992): three merging images (B-A-C; typical of a source lying close to a cusp caustic) face the faint saddle-point image $\mathrm{D}$ lying close to the lensing galaxy G (Fig. 1).

J1131 has several observational advantages compared to the other lens systems, namely an integrated magnitude $R \sim 15.3$ and a large angular separation between the lensed images $(\Delta \theta \sim$ $\left.3.6^{\prime \prime}\right)$. These characteristics make J1131 an ideal target for spectroscopic studies (e.g. Sheinis 2006) and optical flux monitoring

* Based on observations collected at the European Southern Observatory, Paranal, Chile (ESO Program 71.A-0407(B, E)).

$\star \star$ Maître de recherches du FNRS (Belgique).

$\star \star \star$ Directeur de recherches honoraire du FNRS (Belgique). (e.g. Morgan et al. 2006; COSMOGRAIL project). Additionally, the low redshift of the source quasar enables one to observe the $\mathrm{Mg}$ II, $\mathrm{H} \beta$ and $[\mathrm{O}$ III] $\lambda \lambda 49959,5007$ emission lines in the optical range. This allows the comparison of the flux ratios in three different emitting regions, namely the AGN continuum, the broad emission lines (e.g. $\mathrm{H} \beta$ ) and the narrow emission lines (e.g. [O III] $\lambda \lambda 4959,5007)$. Because of their different angular sizes, these three emitting regions will not be similarly affected by micro-lensing due to stars in the lensing galaxy or milli-lensing by more massive substructures (typically $M>$ $\left.10^{4} M_{\odot}\right)$. Therefore, the comparison of the flux ratio measured in those three regions may offer a robust diagnostic of micro/millilensing at work in the system.

Although milli-lensing had been early suspected in J1131 (e.g. Keeton et al. 2003), Sluse et al. (2006, Paper I) gave stronger evidence for the presence of micro-lensing, on the basis of a multi-wavelength study of the flux ratios observed at different epochs. Even when tentatively corrected for micro-lensing, the observed flux ratios were still impossible to reproduce with simple smooth lens models. Claeskens et al. (2006) showed in 


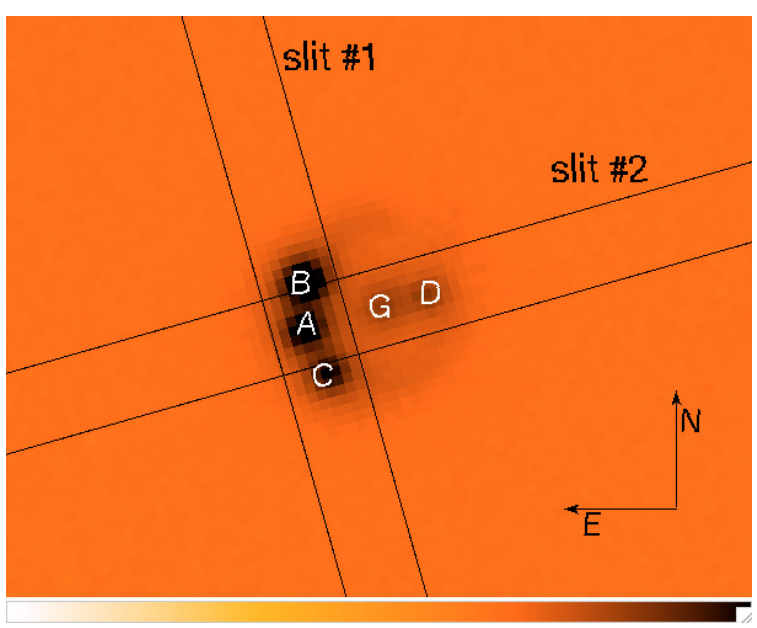

Fig. 1. Image of J1131 (0.8"seeing) with superimposed slit orientations $\# 1$ and \#2. Identification of the quasar lensed images (A, B, C, D) and of the lensing galaxy $(\mathrm{G})$ are also reported.

Paper II that multipole-like models could formally solve the anomaly. For this purpose, they constructed lens models constrained by the relative astrometry of the QSO images provided by the HST images and by substructures present in the nearly complete Einstein ring. This also enabled them to reconstruct the host spiral galaxy of the source AGN and to study in detail its properties. In the present paper, we take advantage of long slit spectroscopy of J1131 to further study micro-lensing in this system and phenomenologically describe its effect on different emitting regions and in particular on the broad line emitting region (BLR).

Abajas et al. (2002) have shown, using the Kaspi et al. (2000) relation linking the BLR size to the quasar luminosity, that the BLR of less luminous quasars is likely small enough (i.e. a few micro-arcseconds) to be affected by micro-lensing. Because the source in J1131 is rather a (bright) Seyfert than a luminous quasar, one may thus expect to observe differential microlensing of its BLR. Thanks to the large spectral coverage of our data (i.e. $2400<\lambda<6900 \AA$; rest frame) we looked for microlensing of the BLR and compared its effect on the main Balmer lines and on the Mg II line. Additionally, we compared the flux ratios in these lines to those in several narrow emission lines (NELs), namely [Ne V], [O II] and [O III]. Finally, we studied micro-lensing of the blended Fe II emission all along the spectra. The location of the Fe II emitting region is still hotly debated (e.g. Zhang et al. 2006b) and micro-lensing of the latter offers the opportunity to compare the size of the Fe II emitting region with the BLR size.

The structure of the paper is the following. We describe the observations and the spectrum extraction in Sect. 2. We perform a preliminary analysis to explore the data in Sect. 3 and provide more quantitative measurements of the spectral differences by means of a multicomponent spectral decomposition in Sect. 4. We propose a comprehensive micro-lensing scenario in Sect. 5 and discuss the implications of the differential micro-lensing of the broad emission lines (BELs) in Sect. 6. We present some additional results concerning the extended nature of the NELs, the absorption systems observed in the QSO spectra in Sect. 7 together with the redshifts of the lens and of two neighbour galaxies. We comment on some working assumptions in Sect. 8 and summarize the results in Sect. 9.
Table 1. Log of the observations for slit orientations \#1 and \#2 (Fig 1). Col. 1: Observing date; Col. 2: Instrument; Col. 3: Total exposure time; Col. 4: Mean seeing during the observations; Col. 5: Mean airmass $\sec (z)$.

\begin{tabular}{lcccc}
\hline \hline $\begin{array}{l}\text { Date } \\
\text { (dd-mm-yy) }\end{array}$ & Instrument & $\begin{array}{c}\text { Exp } \\
(\mathrm{s})\end{array}$ & $\begin{array}{c}\text { seeing } \\
\left({ }^{\prime \prime}\right)\end{array}$ & $\sec (z)$ \\
\hline \multicolumn{5}{c}{ slit \#1 } \\
\hline $26-04-03$ & FORS2 (GR600I) & $4 \times 240$ & 0.55 & 1.33 \\
$26-04-03$ & FORS2 (GR600B) & $4 \times 240$ & $0.4-0.7$ & 1.45 \\
$13-04-03$ & ISAAC (LR+SZ) & $6 \times 200$ & $0.45-0.62$ & 1.19 \\
\hline \multicolumn{5}{c}{ slit \#2 } \\
\hline $26-04-03$ & FORS2 (GR600I) & $3 \times 430$ & 0.53 & 1.07 \\
$26-04-03$ & FORS2 (GR600B) & $3 \times 430$ & $0.6-0.8$ & 1.12 \\
$13-04-03$ & ISAAC (LR+SZ) & $14 \times 200$ & $0.56-0.89$ & 1.40 \\
\hline
\end{tabular}

Unless explicitly stated, we adopt $H_{0}=70 \mathrm{~km} \mathrm{~s}^{-1} \mathrm{Mpc}^{-1}$, $\Omega_{0}=0.3$ and $\Lambda_{0}=0.7$; all the $\chi^{2}$ values reported are reduced values.

\section{Observational overview}

\subsection{Data}

We obtained long slit spectra of J1131 with the FORS2 instrument mounted on the Kueyen (UT2) ESO Very Large Telescope on April 26th 2003. These data consist of two series of spectra with the $1^{\prime \prime}$ slit oriented along the J1131 lensed images BA-C and A-D respectively (hereafter orientations \#1 and \#2; see Fig 1). The Longitudinal Atmospheric Dispersion Corrector (Avila et al. 1997) has enabled us to keep the slit centering within $\sim 0.2^{\prime \prime}$ all along the wavelength range, reducing the slit losses in the blue range. The grisms GR600B and GR600I+OG590 blocking filter have been used, allowing to cover the wavelength ranges $3890<\lambda<6280 \AA$ and $6760<\lambda<8810 \AA$ with a resolving power around 800 and 1500 at the central wavelength. The CCD camera (SR collimator) was unbinned $\left(0.063^{\prime \prime} / \mathrm{pix}\right.$ in the spatial direction) in order to ease the spatial deblending of the spectra. Long slit spectra (with slit orientations identical to FORS spectra) have also been obtained in the near infrared range $(9810<\lambda<11390 \AA$ ) $)$ with the ISAAC instrument placed at the focus of the VLT UT1 telescope (Antu), 13 days before the FORS spectra. The low resolution grating combined with a $1^{\prime \prime}$ slit and a SZ filter leads to a resolving power of 550. Table 1 gives a short log of the observations.

\subsection{Reduction}

The FORS spectra were bias subtracted and flatfielded using IRAF $^{1}$. A set of 5 dome flat fields were used. The wavelength dependent structure of the flat fields was corrected by fitting a high order cubic spline with the response task. The resulting flat field is of sufficient quality to correct the fringing appearing on the raw data. The wavelength calibration was performed thanks to $\mathrm{He}-\mathrm{Hg}-\mathrm{Cd}$ and $\mathrm{He}-\mathrm{Ar}-\mathrm{Ne}$ calibration arcs. Since images $\mathrm{B}$ and $\mathrm{C}$ are not well centered in the slit, we shifted the zeropoint of their wavelength calibrated spectra by $\sim 0.8 \AA$ with respect to A. Finally, the sky background was removed by fitting and subtracting a first order Chebychshev polynomial in the

1 IRAF is distributed by the National Optical Astronomy Observatories (NOAO), which are operated by the AURA, Inc., under cooperative agreement with the National Science Foundation (NSF). 


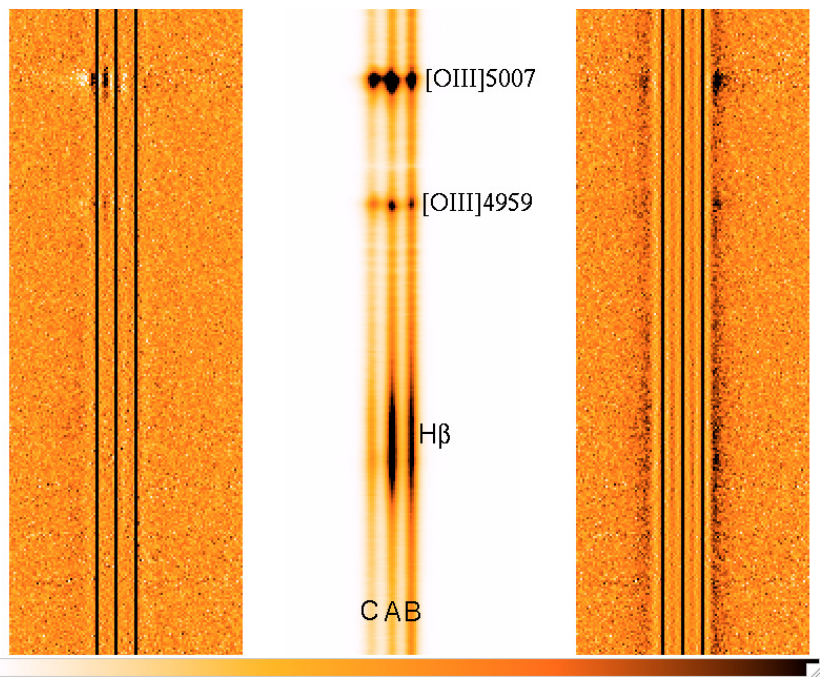

Fig. 2. Central panel: bidimensional Moffat models of the spectra of $\mathrm{C}$, $\mathrm{A}$ and $\mathrm{B}$ in the $\mathrm{H} \beta \lambda 4861 \&[\mathrm{O} \mathrm{III}] \lambda \lambda 4959,5007$ region. Left and right panels: bidimensional residuals $( \pm 3 \sigma)$ for a Moffat and a Gaussian fit. Vertical black lines indicate the center of the profiles for C, A, B.

spatial direction in two adjacent regions of 180 pixels width not illuminated by the object. Since no standard star was observed during the science night, the sensitivity curve was constructed using the two standard stars GD108 (for the GR600B grism) and LTT7379 (for the GR600I grism) observed on April 15th 2003 and May 25th 2003, respectively, through a $2.5^{\prime \prime}$ width slit. The same reduction procedure was applied to these stars, using calibration data associated with these observing dates. The stars and the objects were corrected for sky extinction with the Paranal extinction estimates available on the ESO website.

For the ISAAC spectra, as is standard practice in the infrared, the object was observed in two positions along the slit. The strong and highly variable night sky emission lines were effectively removed by subtracting the resulting spectra from each other. The 2D sky-subtracted spectra were then flat-fielded, registered, wavelength calibrated and co-added. The wavelength calibration was performed using both xenon argon arc lines and OH sky lines (Rousselot et al. 2000). The slit curvature correction was applied using the fitcoords and transform IRAF tasks. Once corrected for the slit curvature, the NIR spectra were still inclined with respect to the pixel grid. We thus applied an additional rotation of $0.78^{\circ}$ to align the spectra with the pixel grid and ease the subsequent spectrum extraction (Sect. 2.3). Finally, the response curve was calculated by dividing the reduced spectrum of the B2 type standard star HIP88126 by a black body at $20000 \mathrm{~K}$. For both optical and NIR data, a noise image was constructed at each step of the reduction, using the standard error propagation formula.

\subsection{Spectrum extraction}

Our extraction method is different for slit orientations \#1 and \#2. For frames obtained with slit orientation \#1, only the point-like images A, B and C were in the slit. We thus fitted three Moffat profiles in the spatial direction for each wavelength bin independently, allowing both the centroid of the Moffat, the slope $\beta$ and $F W H M$ to vary. After this preliminary fit of the bi-dimensional spectra, we smoothed the fitted centroid positions of A-B-C with a $10 \AA$ moving average and reprocessed the extraction by fixing the positions to the ones found after smoothing. The fitted 2D spectra were then compared to the observed ones in order to track for extraction artifacts. Figure 2 compares the residuals observed in the $\mathrm{H} \beta$ and $[\mathrm{O} \mathrm{III}]$ region (i.e. $7900<\lambda<8350 \AA$ ) when extraction is performed with 3 Moffat (left panel) and with 3 Gaussian (right panel) profiles. One can see systematic residuals next to each lensed image spectrum when Gaussian profiles are used. These residuals are indeed observed all along the spectra. On the other hand, the residuals are flat at all wavelengths when Moffat profiles are used, except around [O III] $\lambda \lambda 4959$, 5007. In order to explain the origin of those residuals we constructed a $2 \mathrm{D}$ pseudo-continuum under [O III] $\lambda \lambda 4959,5007$ by interpolating the continuum level measured just before and after [O III]. Once that pseudo-continuum had been subtracted from the data, we obtained 2D spectra of [O III] emission only. The fit of the latter with 3 Moffat profiles is significantly worse than the fit of the same region for the continuum only $\left(\chi^{2}=1.01\right.$ for the continuum and $\chi^{2}=3.71$ for [O III] $\left.\lambda \lambda 4959,5007\right)$. The results are even worse if we impose the Moffat parameters for [O III] to be equal to those found for the continuum $\left(\chi^{2}=4.68\right)$. This is a hint that the observed [O III] $\lambda \lambda 4959,5007$ emission is not point-like but is partially resolved. The complete extracted spectra of A, B and C are displayed in Fig. 3. The flux "lost" in the [O III] region due to imperfect extraction is only $0.5 \%$ of the $[\mathrm{O}$ III $] \lambda \lambda 4959,5007$ emission in $\mathrm{A}+\mathrm{B}+\mathrm{C}$. Therefore, our extraction method does not introduce significant error on the flux measurement in [O III] due to its spatial extension.

After extraction, we constructed a synthetic optical spectrum (sampled on a pixel grid of $1.33 \AA$ ) of each lensed image by joining the blue (GR600B) and red (GR600I) spectra on a common grid. Since there is no overlapping domain between the blue and red spectra, we assume that the fractional loss of flux is similar in each spectrum such that no relative flux rescaling between blue and red is necessary. We did not attempt to rescale in flux the NIR spectra over the optical ones since these spectra were obtained 13 days later. Although A, B and C are not perfectly aligned along the slit, we assume that the relative flux loss between the lensed images is small. To test this, we extracted the flux in the acquisition image along 3 different rectangular apertures of $0.8,1.0$ and $1.2^{\prime \prime}$ widths, each oriented like the true slit. We then fitted A, B, C with 3 one-dimensional Moffat profiles as we did for the spectrum extraction. Following this procedure we found that the measured flux ratios are independent of the chosen slit width. Additionally, these flux ratios agree within 5\% with the ratios of the spectra multiplied by the $R$-band transmission curve.

For the slit orientation \#2, we performed classical extractions in apertures centered on the lensed images A and D and around G, using the IRAF task apall. Unfortunately, the extracted spectra of D were significantly contaminated by the lensing galaxy while the one of A was likely contaminated by images $\mathrm{B}$ and $\mathrm{C}$. Although we could adapt the size of the extraction aperture to reduce the contamination, this precluded a correct flux calibration for the spectrum of D. For this reason, we do not analyse that image in the remaining. Additionally, three other objects are located in the slit. A star (11:31:55.8, -12:32:16) and two galaxies gal\#1 and \#2 (11:31:55.025, -12:32:13 and 11:31:57.7, $-12: 32: 22$ ) located at 55 and $95^{\prime \prime}$ from the lens. They were extracted through fixed apertures of 10 pixel width. These spectra are analysed separately (Sect. 7.3) from those of the lensed images. The latter are described in the following two sections. 


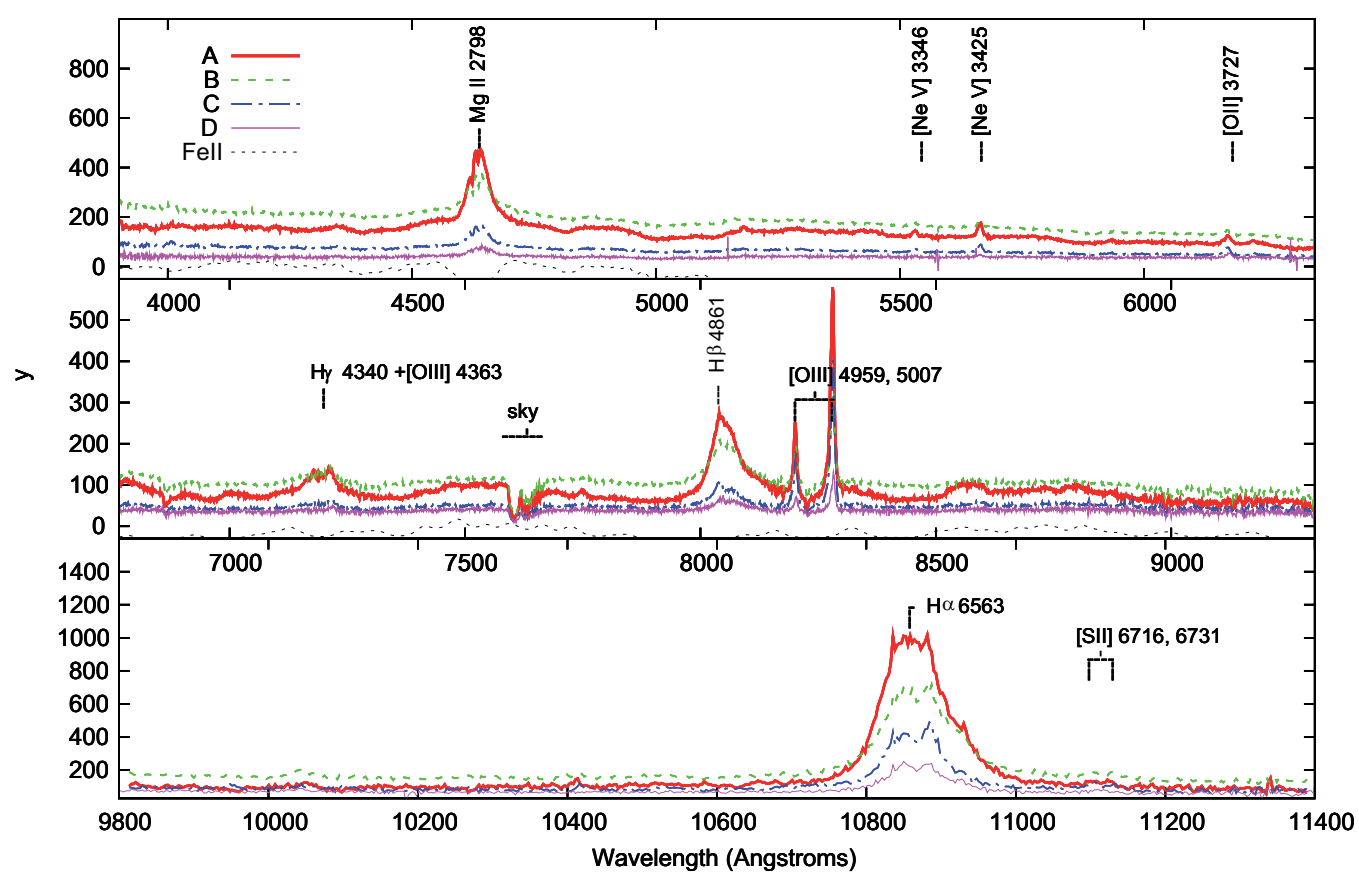

Fig. 3. Extracted spectra of images A (thick solid red), B (dashed green), C (dashed-dotted blue) and D (thin solid pink) in the blue (up), red (middle) and NIR (bottom) ranges. Black dashed line (bottom of the graph) in the blue and red ranges is the template of Fe II emission redshifted at $z=0.657$ and arbitrarily shifted in flux for legibility. Relative flux ratios between the spectra are conserved except for image D. Main emission lines are identified.

\section{Exploratory analysis}

The goal of this section is to identify general features in the AB-C lensed image spectra, by means of a phenomenological and model independent approach. Although numerical results will be obtained, more quantitative results derived from systematic fittings will be presented in Sect. 4.

\subsection{Spectrum ratios}

The first insight into the relative differences between the lensed spectra is simply provided by computing the ratios of individual spectra. Indeed, since gravitational lensing is achromatic, one expects the lensed image spectra to be identical up to a magnification factor. Therefore the spectral ratio between two images is expected to be flat. However, extinction in the lensing galaxy may differently affect the lensed images, as well as micro/milli-lensing produced by substructures in the lens, such that chromatic trends may appear in the spectral ratios (e.g. Jean \& Surdej 1998; Wambsganss \& Paczyński 1991). In some cases, micro/milli-lensing can also modify the profiles of the BELs (e.g. Abajas et al. 2002). The ratio between individual spectra thus provides one with a simple diagnostic of the presence of differential extinction or micro-lensing. The $\mathrm{A} / \mathrm{B}$ and $\mathrm{C} / \mathrm{B}$ ratios are presented in Fig. 4.

One can clearly see the imprint of the QSO emission line spectra in the $\mathrm{A} / \mathrm{B}$ spectral ratio, indicating that the flux ratio is different in the continuum (e.g. 7900-7950 $\AA$ ), the

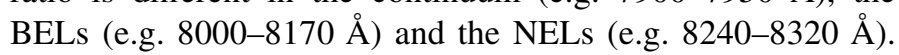
Differences in the pseudo-continuum (i.e. the AGN power law continuum+blended Fe II emission) are also apparent (e.g. 5100-6200 ̊). This suggests that "classic" microlensing is probably at work by affecting the compact region emitting the continuum. However, no chromatic dependence is observed, as illustrated by the flat underlying continuum ratio (tentatively indicated by the dotted line in Fig. 4, at the value $R=0.63$ ).
On the other hand, the $\mathrm{C} / \mathrm{B}$ spectral ratio is not flat. First, most of the BEL structure has disappeared, with the noticeable exception of the $\mathrm{Mg}$ II line, in which the flux ratio is significantly different from the one in the underlying continuum. Second, the flux ratio in the NELs (in particular in [O III] $\lambda \lambda 4959,5007$ ) is drastically different from the one in the continuum (i.e. as measured at the foot of the line "ratio"). Finally, an overall, chromatic trend is observed with a flux ratio increasing from $\sim 0.35$ in the blue to $\sim 0.55$ in the red. An attempt to provide a coherent interpretation with a minimum number of hypotheses is discussed below.

\subsection{The microlensed spectra}

\subsubsection{The $F \mu$ method}

Assuming the observed spectra $F_{i}$ are simply made of a superposition of spectrum $F_{M}$ which is only macro-lensed and of a spectrum $F_{M \mu}$ both macro and micro-lensed, it is easy to extract both components $F_{M}$ and $F_{M \mu}$ by using pairs of observed spectra. However, the relative macro amplification $M$ must be known.

Indeed, defining $M=M_{1} / M_{2}(>0)$ and $\mu=\mu_{1} / \mu_{2}(>0)$ as the constant macro- and micro-amplification ratios between image 1 and image 2, we have:

$F_{1}=M F_{M}+M \mu F_{M \mu}$

$F_{2}=F_{M}+F_{M \mu}$.

The latter equations can be rewritten to extract $F_{M}$ and $F_{M \mu}$ :

$$
\begin{aligned}
F_{M} & =\frac{F_{1} / M-\mu F_{2}}{1-\mu} \\
F_{M \mu} & =\frac{F_{2}-F_{1} / M}{1-\mu},
\end{aligned}
$$

where $\mu$ must be chosen to satisfy the positivity constraint $F_{M}>$ 0 and $F_{M \mu}>0$. 

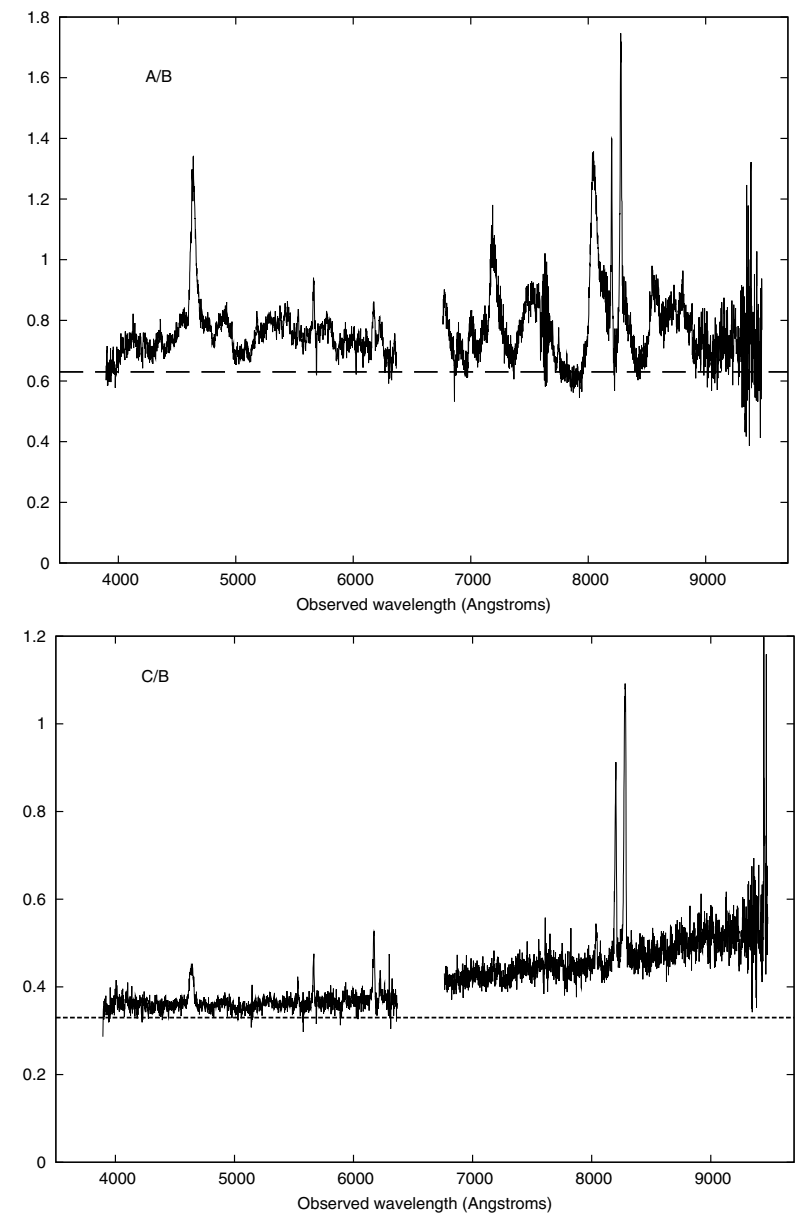

Fig. 4. Ratio of spectra A and B (top) and C and B (bottom). The observed (flat) ratio $R=M \mu$ is plotted with a dotted line. $R=0.63$ for the $\mathrm{A} / \mathrm{B}$ ratio and $R=0.33$ for the $\mathrm{C} / \mathrm{B}$ ratio.

It is clear from Eq. (2) that $F_{M \mu}$ is directly determined from the macro-amplification ratio $M$ and the observed spectra $F_{1}$ and $F_{2}$, up to a scaling factor. On the other hand, if $\mu$ and $\mu^{\prime}$ are two values of the micro-amplification ratio which satisfy the positivity constraints and such that $\mu^{\prime}<\mu$, it is easy to show that:

$F_{M}\left(\mu^{\prime}\right)=F_{M}(\mu)+\frac{\mu-\mu^{\prime}}{1-\mu^{\prime}} F_{M \mu}$.

It is obvious from the previous equation that adopting the smaller value $\mu^{\prime}$ is equivalent to add a fraction of the microlensed spectrum to the macrolensed spectrum obtained with $\mu$. Since an emitting region can only be microlensed or not, the only possible choice for $\mu$ is the maximum value satisfying the positivity constraint.

The previous decomposition is exact when $\mu$ is constant as a function of wavelength, i.e. when the micro-lensing ratio is achromatic. However microlensing is a function of the source size (which is wavelength dependent) and this function can be different for different images, so that even the micro-lensing $r a$ tio $\mu$ can be chromatic. Any such wavelength dependence of $\mu$ would be propagated into the spectra $F_{M}$ and $F_{M \mu}$. Although this is not important as long as the method is simply used to identify spectral features affected by some micro-lensing (i.e. whose flux ratio is not equal to the macro value), we shall show that $\mu=$ constant is a reasonable choice.

In the following, we will call the decomposition of the spectra into $F_{M}-F_{M \mu}$, the $F \mu$ method.

\subsubsection{The A-B pair}

In order to apply the $F \mu$ method to the A-B pair of spectra, the macro amplification ratios must be fixed. In the remainder of the paper, we use the hypothesis that they are given by the observed flux ratios in the $[\mathrm{O}$ III $] \lambda \lambda 4959,5007$ lines. This seems a reasonable choice in the sense that the NELs are produced by the largest emitting region, thus the least affected by microlensing. The fact that the [O III] $\lambda \lambda 4959,5007$ emission is partially resolved, especially between images $\mathrm{C}$ and A (see Sect. 2.3) does not bias the [O III] flux estimate. Indeed, we have shown that only a very small fraction (i.e. $<1 \%$ ) of the flux in [O III] was lost during the extraction (Sect. 2.3).

Under this hypothesis, first we must have that $M=$ $M_{A} / M_{B}=2.1$ in order to suppress the [O III] $\lambda \lambda 4959,5007$ emission lines in the combined spectrum $F_{A}-M F_{B}$. Second, by associating image A (resp. B) with number 1 (resp. 2) in Eqs. (2), we find $\mu=\mu_{A} / \mu_{B}=0.3$. The extracted spectra $F_{M}$ and $F_{M \mu}$ are shown in Fig. 5. Note that a constant value of $\mu$ is certainly a good approximation, given the flat $\mathrm{A} / \mathrm{B}$ underlying continuum (Fig. 4).

The striking result of this decomposition is that, in addition to the continuum, the broadest part of the BEL is also affected by microlensing. This might indicate that at least part of the BLR is very compact. A more thorough discussion is delayed to Sect. 5, after a more quantitative analysis is made in Sect. 4.

On the other hand, as expected, the unaffected spectrum $F_{M}$ is basically flat and consists of the NEL and narrowest part of the BEL. Indeed, not only the [O III] $\lambda \lambda 4959,5007$ lines, but also the core of $\mathrm{Mg}$ II, $\mathrm{H} \gamma$ and $\mathrm{H} \beta$ BELs are prominent. Interestingly, blended Fe II emission is also clearly observed around $\mathrm{Mg}$ II and blue/red-ward of $\mathrm{H} \beta$.

\subsubsection{The B-C pair}

In the case of the B-C pair of spectra, before applying our extraction method, we must first discuss the chromatic trend observed in the ratio spectrum (see Fig. 4). This trend probably comes from image $\mathrm{C}$ since the $\mathrm{A} / \mathrm{B}$ ratio is not affected. It has four possible origins: i- chromatic microlensing; ii- differential extinction along the line-of-sight; iii- host contamination; iv- contamination by the lensing galaxy.

We exclude significant contamination by the lensing galaxy that is about 100 times fainter than image $\mathrm{C}$ at the lensed images position (Paper II). Since none of the other phenomena taken separately is capable of accounting for the observations, we must search for the minimum combination of hypotheses. Indeed, extinction alone is unable to explain the presence of $\mathrm{Mg}$ II in the ratio spectrum and is expected to modify more strongly the spectrum slope in the blue than in the red. Chromatic microlensing is also expected to mainly affect the blue part of the spectrum; finally, the host galaxy is expected to maximize its contamination in the reddest part of the spectrum of the faintest image, as observed, but it is not supposed to produce a differential effect on $\mathrm{Mg}$ II.

Since the host is indeed observed to be quite bright in the red and in the NIR (see Papers I and II), we decided to explore the combined effect of the host contamination together with a wavelength independent microlensing, as modelled in Sect. 3.2.1.

The host contamination probably seen in the spectrum ratio necessarily comes from a constant, resolved contribution (the possibly unresolved host contribution undergoes the same macro-amplification as the QSO and is spatially too large to be microlensed, so that it cannot affect the slope of the spectrum 


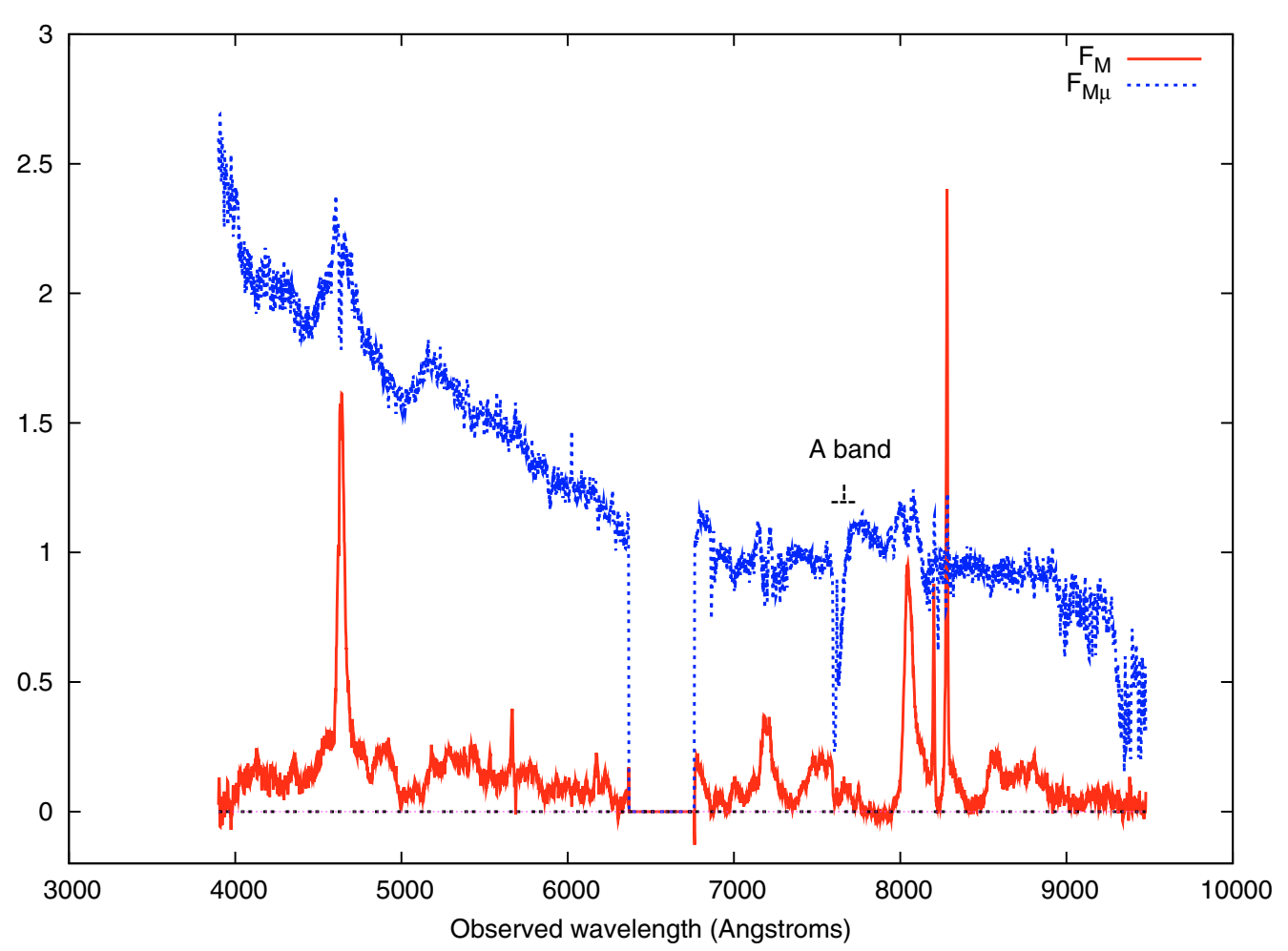

Fig. 5. Fraction of the spectrum affected $\left(F_{M \mu}\right.$, dotted) and unaffected $\left(F_{M}\right.$, solid) by microlensing (arbitrary units) in the A-B spectrum pair (see Eq. (2)). The A-band telluric absorption is also identified.

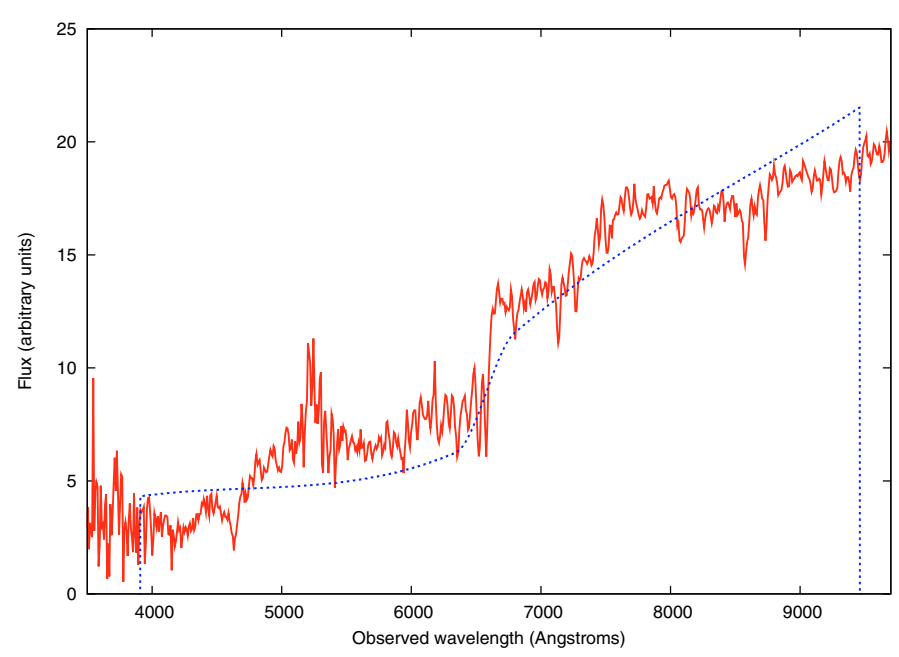

Fig. 6. The smoothed host galaxy spectrum (dotted) responsible for the chromatic trend observed in the $\mathrm{C} / \mathrm{B}$ spectrum ratio, compared with a redshifted spectral template of a Sb galaxy (solid).

ratio). The contamination by such a resolved contribution is additive and, thus, it must first be removed before applying Eqs. (2). Since, conversely, the dominant part of the resolved flux may be associated with the host and the dominant part of the unresolved flux may be associated with the QSO continuum, we can write with a good approximation:

$F_{B}=F_{\mathrm{QSO}}+F_{\text {Host }}$

$F_{C}=R F_{\mathrm{QSO}}+F_{\text {Host }}$,

where $R$ is the intrinsic C/B ratio due to macro- and microamplification. $F_{\text {Host }}$ is obtained after smoothing out residual features in the solution of system 4.
Table 2. Summary of the macro-amplification ratios $M$, microamplification $\mu$ and continuum ratio $R$ values found in the exploratory study.

\begin{tabular}{lcc}
\hline \hline & $\mathrm{A} / \mathrm{B}$ & $\mathrm{C} / \mathrm{B}$ \\
\hline$M$ & 2.1 & 1.4 \\
$\mu$ & 0.30 & 0.25 \\
$R$ & 0.63 & 0.33 \\
\hline
\end{tabular}

Now, the exact choice of the $R$ value is somewhat arbitrary. If the host contamination in the blue is assumed to be negligible, the value $R=0.35$ is chosen, as observed in the blue part of the spectrum ratio (see Fig. 4). A break is seen in the resulting host restframe "spectrum" exactly at the expected position of the Balmer discontinuity, giving a good hint that we indeed deal with the host galaxy (unfortunately the break falls in the gap between the two wavelength ranges covered by our spectra). As a refinement, adopting $R=0.33$ instead of $R=0.35$ adds a slight host contamination in the blue, so that the final contaminating "spectrum" is reminiscent of the spectrum of a Sb galaxy (see Fig. 6). In the first (resp. second) case, the host flux at $5000 \AA$ restframe corresponds to $\sim 50 \%$ (resp. $\sim 75 \%$ ) of the QSO flux observed in image $\mathrm{C}$ at the same wavelength. However, in both cases, the $\mathrm{C} / \mathrm{B}$ spectrum is flattened and no significant difference is observed in the subsequent analysis. Note also that correcting a posteriori for the host contamination in the A-B pair does not significantly affect the results derived in Sect. 3.2.2.

Once the $\mathrm{B}$ and $\mathrm{C}$ spectra are decontaminated (using $R=$ 0.33 ), the spectral decomposition is performed in the same way as for the A-B pair. Associating image C (resp. B) with number 1 (resp. 2) in Eqs. (2), we find $M=M_{\mathrm{C}} / M_{\mathrm{B}}=1.4$ and $\mu=\mu_{\mathrm{C}} / \mu_{\mathrm{B}}=0.25$; the extracted spectra are shown in Fig. 7 . By construction, the unaffected spectrum is flat. The BEL appears 


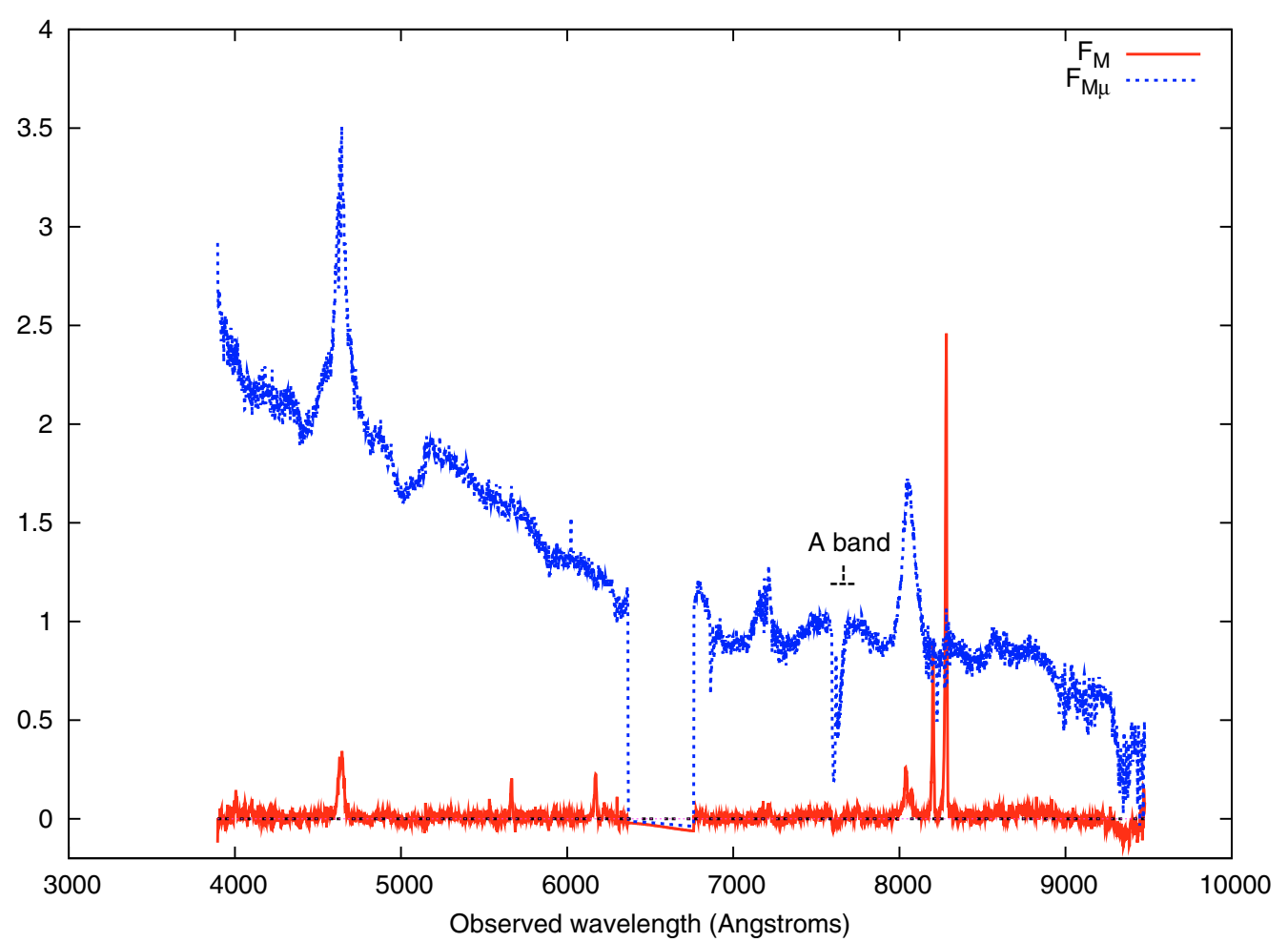

Fig. 7. Fraction of the spectrum affected $\left(F_{M \mu}\right.$, dotted) and unaffected $\left(F_{M}\right.$, solid) by microlensing (arbitrary units) in the B-C spectrum pair, after removing the observed host contamination (see Eq. (2)). The A-band telluric absorption is also identified.

here nearly totally affected by microlensing (except parts of the core of $\mathrm{Mg}$ II and $\mathrm{H} \beta$ BELs). This would confirm that the BLR is very compact. However, as we shall discuss in Sect. 5, this decomposition has to be compared with the one of the A-B pair and interpreted in terms of the micro-lensing of several lensed images.

Before entering into the possible interpretations, we would like to further confirm the previous results by deriving the different flux ratios in a more quantitative way, based on a systematic fitting of the different spectral components (i.e. host, QSOcontinuum, QSO emission lines, ...).

\section{MCD: Multi Component Decomposition analysis}

Examination of our extracted spectra reveals significant emission associated with Fe II. An important fraction of the flux of the host galaxy is also mixed with the flux from the central AGN (Sect. 3.2.3). In order to quantitatively disentangle the different emission components (which might be differently affected by microlensing), we used a Multi-Component Decomposition (MCD) approach (e.g. Wills et al. 1985; Dietrich et al. 2003). This method assumes our rest frame spectra to be a superposition of: (1) a power law continuum $\left(F_{v} \sim v^{\alpha}\right)$, (2) a Balmer emission continuum, (3) a pseudo-continuum due to the merging of Fe II emission blends, (4) a galactic template for the AGN's host galaxy, and (5) an emission spectrum due to the other individual broad emission lines. The fitting of the spectra is a classical least square minimization using a Levenberg-Marquard based algorithm adapted from the Numerical recipes routine (Vetterling et al. 1993). We give hereafter the technical details associated with this method. We use it to decompose the observed spectra of AB-C (Sect. 4.2) and discuss the errors in Sect. 4.3.

\subsection{Overview of the method}

We performed the fit iteratively. First we fitted components (1), (2) and (4) in three rest-frame windows nearly free of Fe II emission, namely $\lambda \lambda 2600-2700 \AA, 2950-3100 \AA$ and 5400-5500 ̊ (Natali et al. 1998). Secondly, we imposed a null weight to the emission line regions and fitted components (1) to (4) using the previous results as initial conditions. Finally we included the emission lines in the fit.

\subsubsection{The quasar continuum}

We modelled the quasar emission as:

$F_{\lambda}=F_{0} \times{\frac{\lambda}{\lambda_{0}}}^{-(2+\alpha)}+F_{\lambda}^{\mathrm{Bac}}$

where $\lambda_{0}=5000 \AA, \alpha$ is the canonical power law index and $F_{\lambda}^{\mathrm{Bac}}$ is the Balmer continuum intensity. To estimate the Balmer continuum from our fits, we have used the empirical distribution by Grandi (1982) which assumes gas clouds of uniform temperature $T_{\mathrm{e}}$ that are partially optically thick. In this case the Balmer continuum spectrum $(\lambda \leq 3646 \AA)$ can be described by:

$F_{\lambda}^{\mathrm{Bac}}=F^{\mathrm{BE}} B_{\lambda}\left(T_{\mathrm{e}}\right)\left(1-\mathrm{e}^{-\tau_{\lambda}}\right), \quad \lambda \leq \lambda_{\mathrm{BE}}$

with $B_{\lambda}\left(T_{\mathrm{e}}\right)$ as the Planck function at the electron temperature $T_{\mathrm{e}}, \tau_{\lambda}$ as the optical depth at $\lambda$, and $F^{\mathrm{BE}}$ as a normalized estimate for the Balmer continuum flux density at the Balmer edge $\lambda_{\mathrm{BE}}=3646 \AA$. At wavelengths $\lambda>\lambda_{\mathrm{BE}}$ higher order Balmer lines are merging into a pseudo continuum, yielding a small rise to the Balmer edge. We did not attempt to model this region and kept $F_{\lambda}^{\text {Bac }}=0$ at $4150 \AA>\lambda>\lambda_{\mathrm{BE}}$. In order to ease the minimization, we fixed the electron temperature and the optical depth 
$\tau_{\lambda}$ to realistic values (i.e. $\tau_{\lambda}=2.0$ and $T_{\mathrm{e}}=15000 \mathrm{~K}$; Wills et al. 1985; Dietrich et al. 2003). The region 3080-3540 $\AA$ is imperfectly modelled using Eq. (6). We observe a significant excess of emission w.r.t. the model. This one is likely explained by blended Fe II emission for which there is presently no available template and which are poorly reproduced by photo-ionization models (e.g. Véron-Cetty et al. 2006). We thus excluded this region from the fitting procedure.

\subsubsection{The Fell emission}

Following many authors (e.g. Wills et al. 1985; Laor et al. 1997; Dietrich et al. 2003; Tsuzuki et al. 2006), we used template spectra of Fe II emission instead of theoretical emission models. Namely, we fitted the Fe II emission in our spectra by using an UV Fe II template (Vestergaard \& Wilkes 2001) and an optical Fe II template (Véron-Cetty et al. 2004). We also tested the optical template of Boroson \& Green (1992) but this did not modify significantly our results. We prefered the optical template of Véron-Cetty et al. especially due to its larger spectral coverage. Since UV photons are generally absorbed and re-emitted at optical wavelengths (for high optical depth), optical and UV Fe II emission are often anti-correlated (Joly M., private communication). For this reason, we have not fixed the intensity ratio between the UV and optical Fe II templates. The templates have been broadened in the log space (in order to keep the velocity width constant over the whole wavelength domain) using the $F W H M$ of the $\mathrm{H} \beta$ emission line. The broadened templates are shown in Fig. 3.

For technical reasons, in the Fe IIUV template of Vestergaard $\&$ Wilkes, the Fe II level beneath the central part of the Mg II emission (i.e. $2787<\lambda<2802 \AA$ ) is 0 . We have thus constructed a modified Fe II template for which we consider a non zero Fe II level between $2752<\lambda<2832 \AA$. We estimate this level by interpolating in the template the Fe II level between the two closest maxima enclosing the zero Fe II hole. These maxima are found at 2752 and $2832 \AA$. Both templates have been used during our decomposition. The effect of this modified template on the results is discussed in Sect. 4.2.2.

\subsubsection{Host galaxy}

We used a Sb galactic template (Kinney 1993) to model the host galaxy. The latter choice is motivated by the detailed study of the host performed in Paper II. It is also supported by the empirical host spectrum we have retrieved in Sect. 3 and which has a continuum emission compatible with a Sb type galaxy (Fig. 6).

\subsubsection{Emission lines}

During the last iteration of our fitting procedure, the emission lines were fitted simultaneously with the other components as a sum of Gaussian profiles. A minimum of two profiles was considered for the main emission lines. More details are given in Sect. 4.2.2.

\subsection{Application to $A-B-C$ spectra}

We present in this section the results of the MCD method applied to the spectra of images $\mathrm{A}, \mathrm{B}$ and $\mathrm{C}$ in order to estimate the flux ratios in the different emission regions. Unfortunately, we found that there is a degeneracy between three quantities: the host galaxy relative flux, the power law index $\alpha$ and the Balmer continuum level $F_{\lambda}^{\text {Bac }}$. Due to the large range of acceptable values for $\alpha$ implied by the degeneracy, we could not disentangle the host galaxy emission from the QSO emission and we could not assess the presence (absence) of chromatic variation of the quasar continuum (due either to microlensing or differential extinction). In the following, we thus decide to make a prior on $\alpha$ in order to break the degeneracy. This has no major effects on our conclusions as explained in Sect. 4.3.

Motivated by the results of Sect. 3, we imposed $\alpha$ to be identical for each image. We choose $\alpha=-0.125$ which is at the middle of the overlapping range of acceptable values for $\alpha$ in $\mathrm{A}, \mathrm{B}, \mathrm{C}$, when no prior is imposed. This value is also similar to the best value found for image $\mathrm{B}$. This value is in good agreement with the mean power law index $\alpha=-0.33 \pm 0.59$ found by Natali et al. (1998) in the range 1200-5500 $\AA$ or with the power law index of the composite SDSS quasar spectra measured in the range $1300-5000 \AA$ (i.e. $\alpha=-0.44$; Vanden Berk et al. 2001). Results are given in Table 3 .

\subsubsection{The continuum}

The flux ratios between the power law continua are directly obtained from the MCD results (Table 3 ) and are reported in Table 5. We find $F_{\mathrm{A}} / F_{\mathrm{B}}=0.55 \pm 0.02$ and $F_{\mathrm{C}} / F_{\mathrm{B}}=0.31 \pm 0.01$, in good agreement with the rough measurements performed on the direct spectral ratios $\left(F_{\mathrm{A}} / F_{\mathrm{B}}=0.63\right.$ and $F_{\mathrm{C}} / F_{\mathrm{B}}=0.33$, see Table 2). Since the estimate of the host galaxy contribution to the observed spectra is very different with both methods (i.e. $180 \%$ of the QSO flux in C from the MCD method and 75\% from the direct ratio), we are confident that the error on the host galaxy level does not dramatically bias our continua flux ratios ${ }^{2}$.

\subsubsection{The emission lines}

Faint NELs were fitted with a single Gaussian ${ }^{3}$ profile while the bright [O III] $\lambda \lambda 4959,5007$ were fitted with 2 components. The latter decomposition enables us to take into account the line asymmetry of the [O III $] \lambda \lambda 4959,5007$ emission lines. Since the FWHM of the narrowest component is close to the spectral resolution, one should not over-interpret this decomposition. In the following, we will not use the flux measured in the individual components but rather the total flux in the line.

Regarding the BELs, as it is a common practice, we decomposed each line into two broad Gaussian profiles (e.g. VéronCetty \& Véron 2000; Sulentic et al. 2000; Romano et al. 1996) and we added a narrow component when necessary. We enforced the $F W H M$ and $\lambda_{\mathrm{c}}$ of the various sub-profiles to be identical for each lensed image. Motivated by the claimed similarity between the $\mathrm{Mg}$ II and Balmer line profiles (e.g. Mc Lure $\&$ Jarvis 2002), we enforced the velocity width of the 2 broad components (i.e. $F W H M \sim 2000$ and $4300 \mathrm{~km} \mathrm{~s}^{-1}$ ) to be identical - within $50 \mathrm{~km} \mathrm{~s}^{-1}$ - for each broad line. However, like e.g. Corbett et al. (2003), we found necessary to add a very broad component $\left(F W H M \sim 22000 \mathrm{~km} \mathrm{~s}^{-1}\right)$ to the $\mathrm{Mg}$ II profile. We did not find any evidence for such a component in the Balmer line profile. We supposed that the different decomposition found for Mg II could be due to the hole of Fe II beneath Mg II in the

\footnotetext{
2 Note that it neither affects the $F \mu$ decomposition of the spectra. Namely, a similar $F \mu$ decomposition is obtained with these two different host estimates.

3 Due to the low contrast between [Ne IV] $\lambda 2419$ and $\mathrm{H} \gamma+[\mathrm{O}$ III $] \lambda \lambda 4340,4363$ emissions w.r.t. their underlying continuum, the fit of those lines is inaccurate and not presented.
} 
Table 3. Multi-component best fit of the spectra when $\alpha$ is forced to be identical in A, B, C. Column 1 is the image ID, Col. 2 is the power law index $\alpha$ of the quasar continuum, Col. 3 gives the flux level of the power law continuum at $\lambda=5000 \AA$ (rest-frame), Col. 4 gives the flux level of the Balmer continuum, Col. 5 gives the relative flux of the host galaxy at $5000 \AA$ normalized by $F_{0}^{\mathrm{PWL}}$ (in B), Col. 6 is the level of the optical template of Fe II, Col. 7 is the level of the UV template of Fe II. All the quantities (except $\alpha$ ) are normalized to image B.

\begin{tabular}{ccccccc}
\hline \hline image & $\alpha$ & $F_{0}^{\text {PWL }}$ & $F^{\text {Bac }}$ & $F_{\text {host }, 0}$ & Fe II & Fet \\
\hline A & -0.125 & $0.55 \pm 0.02$ & $0.94 \pm 0.09$ & $0.58 \pm 0.02$ & $1.91 \pm 0.03$ & $2.29 \pm 0.08$ \\
B & -0.125 & $1.00 \pm 0.01$ & $1.00 \pm 0.09$ & $0.77 \pm 0.02$ & $1.00 \pm 0.03$ & $1.00 \pm 0.08$ \\
C & -0.125 & $0.31 \pm 0.01$ & $0.41 \pm 0.09$ & $0.54 \pm 0.01$ & $0.43 \pm 0.03$ & $0.82 \pm 0.06$ \\
\hline
\end{tabular}

Table 4. Multi-component fit of the main emission lines normalized to the total flux in $\mathrm{H} \beta$ for image A. Note that $\mathrm{H} \alpha$ normalization is arbitrary due to the wrong flux scaling of the NIR spectra of J1131 w.r.t. the optical spectra.

\begin{tabular}{ll|cccccc}
\hline & ID & $\lambda_{\mathrm{c}}$ & $F W H M$ & $F_{\mathrm{A}}$ & $F_{\mathrm{B}}$ & $F_{\mathrm{C}}$ & $z$ \\
\hline $\mathrm{Mg}$ II $\lambda 2798$ & BC1 & 4636.4 & 2050 & $0.281 \pm 0.006$ & $0.077 \pm 0.006$ & $0.081 \pm 0.004$ & 0.657 \\
& BC2 & 4638.4 & 4320 & $0.867 \pm 0.009$ & $0.557 \pm 0.008$ & $0.251 \pm 0.006$ & 0.658 \\
& VBC & 4629.4 & 21830 & $0.728 \pm 0.013$ & $0.646 \pm 0.009$ & $0.215 \pm 0.006$ & 0.655 \\
& TOT & & & $1.842 \pm 0.017$ & $1.253 \pm 0.014$ & $0.534 \pm 0.009$ & \\
$\mathrm{H} \beta \lambda 4861$ & no VBC & & & $1.148 \pm 0.010$ & $0.634 \pm 0.010$ & $0.332 \pm 0.007$ & \\
& NC1 & 8039.6 & 460 & $0.040 \pm 0.001$ & $0.012 \pm 0.001$ & $0.016 \pm 0.001$ & 0.654 \\
& BC1 & 8054.2 & 2000 & $0.345 \pm 0.005$ & $0.134 \pm 0.005$ & $0.091 \pm 0.004$ & 0.657 \\
& BC2 & 8055.4 & 4310 & $0.615 \pm 0.006$ & $0.477 \pm 0.006$ & $0.159 \pm 0.005$ & 0.657 \\
& TOT & & & $1.000 \pm 0.008$ & $0.623 \pm 0.008$ & $0.266 \pm 0.006$ & \\
H $\alpha \lambda 6562$ & BC & & & $0.960 \pm 0.008$ & $0.611 \pm 0.008$ & $0.250 \pm 0.006$ & \\
& NC1 & 10850.8 & 550 & $0.496 \pm 0.015$ & $0.242 \pm 0.015$ & $0.276 \pm 0.015$ & 0.653 \\
& NC2 & 10831.7 & 550 & $0.591 \pm 0.013$ & $0.244 \pm 0.013$ & $0.293 \pm 0.013$ & 0.654 \\
& NC3 & 10884.8 & 550 & $0.199 \pm 0.018$ & $0.158 \pm 0.018$ & $0.337 \pm 0.018$ & 0.653 \\
& BC1 & 10874.5 & 2070 & $3.595 \pm 0.063$ & $1.686 \pm 0.063$ & $1.122 \pm 0.063$ & 0.657 \\
& BC2 & 10872.5 & 4300 & $5.480 \pm 0.078$ & $4.227 \pm 0.079$ & $1.533 \pm 0.080$ & 0.657 \\
& TOT & & & $10.361 \pm 0.104$ & $6.557 \pm 0.104$ & $3.562 \pm 0.105$ & \\
{$[\mathrm{O} \mathrm{III}] \lambda \lambda 4959,5007$} & BC & & & $9.075 \pm 0.101$ & $5.913 \pm 0.101$ & $2.655 \pm 0.102$ & \\
& & & & & & & \\
& NC1 & 8202.1 & 240 & $0.052 \pm 0.001$ & $0.031 \pm 0.001$ & $0.038 \pm 0.001$ & 0.654 \\
& NC2 & 8198.8 & 660 & $0.073 \pm 0.002$ & $0.034 \pm 0.002$ & $0.049 \pm 0.002$ & 0.653 \\
& NC3 & 8280.7 & 310 & $0.214 \pm 0.002$ & $0.110 \pm 0.001$ & $0.150 \pm 0.002$ & 0.654 \\
{$[\mathrm{Ne}$ V $] \lambda 3425$} & NC4 & 8275.6 & 800 & $0.172 \pm 0.003$ & $0.084 \pm 0.002$ & $0.108 \pm 0.002$ & 0.653 \\
& TOT & & & $0.511 \pm 0.004$ & $0.259 \pm 0.003$ & $0.345 \pm 0.003$ & \\
& NC1 & 5664.3 & 520 & $0.033 \pm 0.002$ & $0.017 \pm 0.002$ & $0.019 \pm 0.001$ & 0.653 \\
& NC1 & 6171.6 & 620 & $0.023 \pm 0.001$ & $0.013 \pm 0.001$ & $0.023 \pm 0.001$ & 0.656 \\
\hline
\end{tabular}

Fe IIUV template. However, the use of a modified Fe II template in which we "filled" the Fe II hole (Sect. 4.1.2) does not prevent the presence of a very broad component in the $\mathrm{Mg}$ II profile. The fit of Mg II was even found to be worse with this modified template and especially, the peak of the $\mathrm{Mg}$ II emission could not be fitted correctly anymore. Finally, we modelled the faint $\mathrm{Mg}$ II narrow absorption line doublet seen in the $\mathrm{Mg}$ II broad emission with 2 Gaussian profiles separated by $7.2 \AA$ rest frame (Sect. 7.2).

For images B and C, our best fitted profile of the broad emission lines was indistinguishable (in terms of $\Delta \chi^{2}$ ) from the profile obtained when no prior was imposed on the decomposition. For image A, when no prior was imposed on the fit, we found that the best decomposition of the Balmer lines was made of two broad profiles separated by typically $1000 \mathrm{~km} \mathrm{~s}^{-1}$ suggesting some asymmetry in the line profile.

We report in Table 4 the results of the fits performed with the MCD method. Figure 8 shows the continuum subtracted emission lines in each lensed image, together with their modelled profile.

The flux ratios calculated using the results of Table 4 are similar for $\mathrm{H} \alpha, \mathrm{H} \beta \& \mathrm{Mg}$ II BELs. The flux ratios calculated for the narrow [O III] $\lambda \lambda 4959,5007 \&[\mathrm{Ne} \mathrm{V}] \lambda 3425$ emission lines agree within the error bars but are different from those obtained in the BELs. This independently confirms our previous finding that both $F_{\mathrm{A}} / F_{\mathrm{B}}$ and $F_{\mathrm{C}} / F_{\mathrm{B}}$ have different values for the BELs and for the NELs. Note that due to the excellent agreement between the flux ratios measured in [O III] $\lambda 4959(\mathrm{NC} 1+\mathrm{NC} 2)$ and $[\mathrm{O} \mathrm{III}] \lambda 5007$ (NC3+NC4), we do not distinguish between these two lines in the following and rather consider the total flux $[\mathrm{O} \text { III }]_{\text {tot }}$ measured in the $[\mathrm{O}$ III $]$ doublet.

The most precise flux ratio estimates are obtained for the broad component $\mathrm{BC}$ of $\mathrm{H} \beta$ and for $[\mathrm{O} \mathrm{III}]_{\text {tot }}$. They represent characteristic values of the BEL and the NEL respectively and are reported in Table 5. We note that the [O III] flux ratio values $\left(F_{\mathrm{A}} / F_{\mathrm{B}}([\mathrm{O} \mathrm{III}])=1.97\right.$ and $\left.F_{\mathrm{C}} / F_{\mathrm{B}}([\mathrm{O} \mathrm{III}])=1.33\right)$ found here are in good agreement with the macro-amplification ratios derived in Sect. 3 to match the [O III] emission in each spectrum (see Table 2).

Interestingly, for each BEL, the flux ratios found for the broadest components (e.g. BC2 or VBC) are different from those of the narrowest broad component (BC1) except for $F_{\mathrm{A}} / F_{\mathrm{C}}$.

The Fe $\mathrm{II}_{\mathrm{opt}}$ flux ratio (Table 3 ) is similar to the $\mathrm{H} \beta$ ratio for the image pair $\mathrm{B}-\mathrm{C}$ and to the [O III] ratio for the image pair A-B, as can be easily seen in Table 5 . There is an indication for a similar behaviour in Fe IIUV $_{U}$ but the flux ratios deviate more significantly from those obtained in $\mathrm{H} \beta$ and [O III].

Finally, a systemic redshift $z_{\text {systemic }}=0.657 \pm 0.001$ has been determined from the Mg II emission. The measured narrow emission lines are all blue-shifted w.r.t. the systemic redshift 

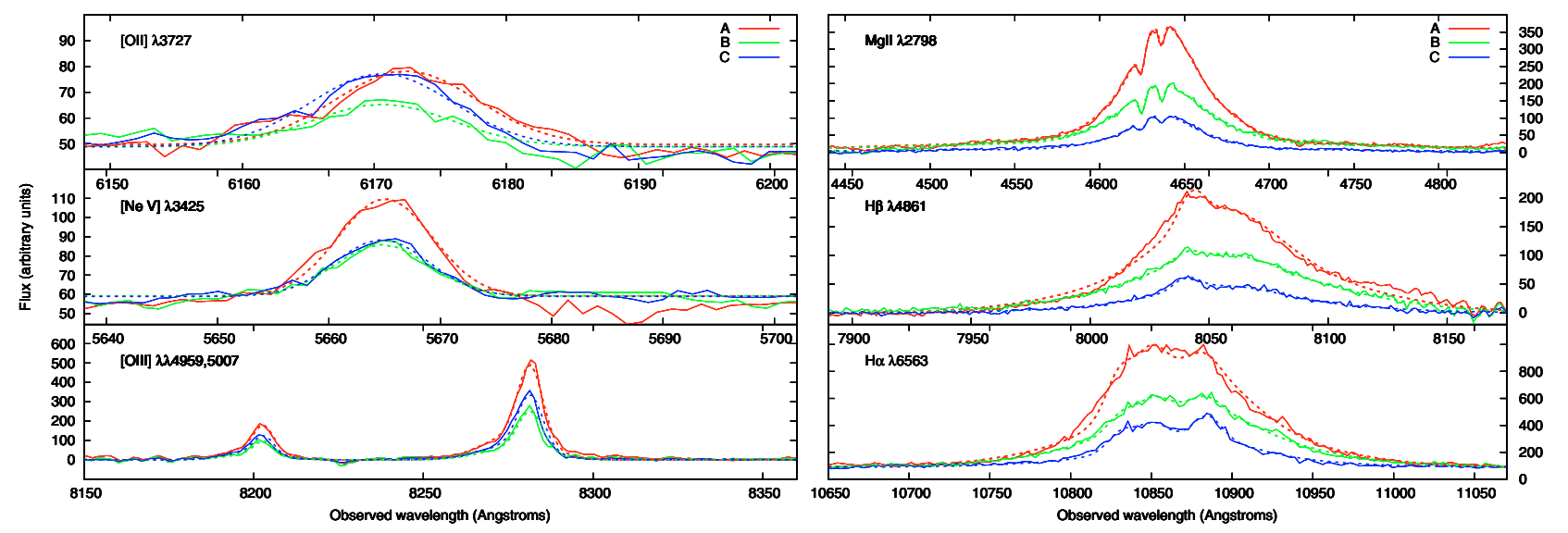

Fig. 8. Comparison of the main NEL (left panel) and BEL (right) for A (solid red), B (solid green) and C (solid blue) with their multi-component fitted model (superimposed with dotted lines). $\mathrm{H} \beta, \mathrm{Mg}$ II and [O III] $\lambda \lambda 4959,5007$ are continuum subtracted profiles. For $\mathrm{H} \alpha$, [Ne V] and [O II] , we have normalized the apparent pseudo continuum to the one of image $\mathrm{C}$.

Table 5. Summary of the flux ratio values measured with the MCD method for several important spectral features.

\begin{tabular}{lcc}
\hline \hline & $\mathrm{A} / \mathrm{B}$ & $\mathrm{C} / \mathrm{B}$ \\
\hline$[\mathrm{O}$ III $]$ & $1.97 \pm 0.03$ & $1.33 \pm 0.02$ \\
$\mathrm{H} \beta$ & $1.57 \pm 0.06$ & $0.41 \pm 0.01$ \\
$\mathrm{Fe}$ II $_{\text {opt }}$ & $1.91 \pm 0.08$ & $0.43 \pm 0.04$ \\
Continuum & $0.55 \pm 0.02$ & $0.31 \pm 0.01$ \\
\hline
\end{tabular}

$\left(z_{\mathrm{NEL}}=0.654 \pm 0.001\right)$ except $[\mathrm{O} \mathrm{II}]$ emission $\left(z_{[\mathrm{OII}}=0.656 \pm\right.$ $0.001)$. We also notice that the red wing of the emission line profile of $[\mathrm{O} \mathrm{II}]$ is significantly more pronounced in image A (Fig. 8), probably because of some underlying Fe II emission (which may deviate from the template we used) and/or to emission associated with star formation located in a direction orthogonal to the slit.

In summary, the MCD analysis performed in this section confirms the main results unveiled in Sect. 3. First, we measure significantly different flux ratios for the continuum emission, the BELs and the NELs. Secondly, we confirm differential magnification of the broadest component of the BELs compared to the core of the emission lines as shown in e.g. Fig. 5. Finally, we find that the flux ratio $F_{\mathrm{C}} / F_{\mathrm{B}}$ (resp. $F_{\mathrm{A}} / F_{\mathrm{B}}$ ) in Fe II is similar to the one of the BEL (resp. NEL).

\subsection{Error analysis}

The MCD method provides us with $1 \sigma$ formal errors calculated from the covariance matrix in the Levenberg-Marquard minimization routine (Vettering et al. 1993). The main limitation of the MCD technique comes from a degeneracy between the power law index $\alpha$ (and the level $F_{0}^{\mathrm{PWL}}$ ), the Balmer continuum emission $\left(F^{\mathrm{Bac}}\right.$ ) and the galactic template contribution. This means that these quantities cannot be retrieved unambiguously. Before fixing the same value for $\alpha$ in each image, we have estimated the range of acceptable values for $\alpha$ and $F_{0}^{\mathrm{PWL}}$ by fixing the host galaxy level to two extreme values beyond which the fit becomes unacceptable. For that range of acceptable continuum spectral decompositions, we found that the fit of the $\mathrm{Mg}$ II, $\mathrm{H} \beta$, [O III] $\lambda \lambda 4959,5007$ and $\mathrm{Fe}_{\mathrm{II}}$ opt remained unchanged. On the other hand, the Fe IIUV intensity was retrieved with only $20 \%$ accuracy. Consequently, imposing the same value for $\alpha$ in each
Table 6. Micro-amplification ratio $\mu_{i} / \mu_{j}$ between images $i$ and $j$ calculated by normalizing the flux ratio measured for a given emission region by the flux ratio measured in [O III] $\lambda \lambda 4959,5007$ (Sect. 5). For completeness, we have also added the micro-amplification ratios computed from optical and X-ray imaging at different epochs ( $\mathrm{mm} / \mathrm{yy})$. Note that $F W H M(B C 1) \sim 2050 \mathrm{~km} \mathrm{~s}^{-1} ; F W H M(B C 2) \sim 4300 \mathrm{~km} \mathrm{~s}^{-1}$; $F W H M(V B C) \sim 22000 \mathrm{~km} \mathrm{~s}^{-1}$.

\begin{tabular}{|c|c|c|c|c|}
\hline \multicolumn{2}{|l|}{ ID } & $\mu_{\mathrm{A}} / \mu_{\mathrm{B}}$ & $\mu_{\mathrm{A}} / \mu_{\mathrm{C}}$ & $\mu_{\mathrm{C}} / \mu_{\mathrm{B}}$ \\
\hline \multicolumn{2}{|c|}{ [O III] $\lambda \lambda 4959,5007_{\text {tot }}$} & $1.00 \pm 0.02$ & $1.00 \pm 0.03$ & $1.00 \pm 0.02$ \\
\hline \multicolumn{2}{|c|}{$[\mathrm{Ne} \mathrm{V}] \lambda 3425$} & $0.97 \pm 0.10$ & $1.16 \pm 0.13$ & $0.83 \pm 0.11$ \\
\hline \multicolumn{2}{|c|}{ [O II] $] \lambda 3727$} & $0.88 \pm 0.11$ & $0.68 \pm 0.08$ & $1.29 \pm 0.12$ \\
\hline \multicolumn{2}{|c|}{ Mg II $\lambda 2798$ BC1 } & $1.85 \pm 0.14$ & $2.33 \pm 0.21$ & $0.79 \pm 0.09$ \\
\hline & $\mathrm{BC} 2$ & $0.79 \pm 0.02$ & $2.34 \pm 0.08$ & $0.34 \pm 0.03$ \\
\hline & VBC & $0.57 \pm 0.02$ & $2.29 \pm 0.09$ & $0.25 \pm 0.04$ \\
\hline & TOT & $0.75 \pm 0.02$ & $2.33 \pm 0.07$ & $0.32 \pm 0.03$ \\
\hline & no VBC & $0.92 \pm 0.02$ & $2.34 \pm 0.08$ & $0.39 \pm 0.03$ \\
\hline \multirow[t]{5}{*}{$\mathrm{H} \beta \lambda 4861$} & $\mathrm{NC} 1$ & $1.72 \pm 0.21$ & $1.66 \pm 0.23$ & $1.03 \pm 0.14$ \\
\hline & BC1 & $1.31 \pm 0.05$ & $2.56 \pm 0.15$ & $0.51 \pm 0.06$ \\
\hline & BC2 & $0.65 \pm 0.01$ & $2.61 \pm 0.10$ & $0.25 \pm 0.04$ \\
\hline & TOT & $0.82 \pm 0.02$ & $2.54 \pm 0.08$ & $0.32 \pm 0.03$ \\
\hline & BC & $\mathbf{0 . 8 0} \pm \mathbf{0 . 0 2}$ & $2.59 \pm 0.09$ & $\mathbf{0 . 3 1} \pm \mathbf{0 . 0 3}$ \\
\hline \multicolumn{2}{|l|}{$\mathrm{Fe} \mathrm{II}_{\mathrm{opt}}$} & $0.97 \pm 0.04$ & $3.04 \pm 0.28$ & $0.32 \pm 0.10$ \\
\hline \multicolumn{2}{|c|}{$\mathrm{Fe} \mathrm{II}_{\mathrm{UV}}$} & $1.16 \pm 0.12$ & $1.89 \pm 0.18$ & $0.61 \pm 0.12$ \\
\hline \multicolumn{2}{|c|}{ Continuum } & $0.28 \pm 0.01$ & $1.23 \pm 0.07$ & $0.23 \pm 0.04$ \\
\hline \multicolumn{2}{|c|}{$R$ band $04 / 03$} & 0.31 & 1.24 & 0.26 \\
\hline \multicolumn{2}{|c|}{$R$ band $04 / 04^{a}$} & 0.46 & 1.69 & 0.27 \\
\hline \multicolumn{2}{|c|}{$R$ band $04 / 05^{b}$} & 0.58 & 1.43 & 0.41 \\
\hline \multicolumn{2}{|c|}{ X Ray $04 / 04^{c}$} & 0.09 & 0.45 & 0.20 \\
\hline
\end{tabular}

${ }^{a}$ Paper I. ${ }^{b}$ Morgan et al. (2006). ${ }^{c}$ Pooley et al. (2006).

lensed image is safe and does not induce systematic error on the fit of the emission lines (including Fe II).

However, we want to be more cautious regarding the results of the [Ne V] $\lambda 3425$, [O II] $\lambda 3727$ emission lines. Indeed, we have no satisfying model for the pseudo continuum under these lines. Therefore, the Gaussian fitting of these lines is performed independently using a flat pseudo-continuum under the line. Because of the likely presence of Fe II in that pseudo-continuum, our procedure may introduce a systematic bias on the fitted line intensity. 


\section{Towards a microlensing scenario}

In this section we look whether the difference in flux ratios observed in the NELs, the BELs and continuum (Sects. 3 and 4.2) can be understood by means of micro-lensing occurring in one or several lensed images. We first identify a possible scenario following a quantitative approach and then discuss the implications.

\subsection{A possible microlensing scenario}

Because the narrow line emission takes place in a region larger than the BLR and the continuum, it is likely to be much less affected by micro-lensing. Therefore, as already mentioned in Sect. 3, we can reasonably assume that the macro-amplification ratios are close to the flux $\operatorname{ratios}^{4}$ in $[\mathrm{O}$ III] $\lambda \lambda 4959,5007$. So, we can write:

$\frac{M_{i}}{M_{j}}=\frac{F_{i}}{F_{j}}\left(\left[\mathrm{O}_{\mathrm{III}}\right]\right)$

where $M_{i}\left(M_{j}\right)$ is the macro-amplification of image $i(j)$ and $F_{i} / F_{j}$ is the flux ratio in [O III] calculated for images $i$ and $j$. On the other hand, if micro-lensing $\mu_{i}\left(\mu_{j}\right)$ occurs in image $i(j)$, we can write for a given emission region:

$\frac{F_{i}}{F_{j}}($ emission $)=\frac{\mu_{i}}{\mu_{j}} \frac{M_{i}}{M_{j}}$.

Consequently, if we normalize the flux ratio estimated for a given emission region by the flux ratio in [O III] $\lambda \lambda 4959,5007$, we infer the micro-amplification ratio $\mu_{i} / \mu_{j}$ for that region. Table 6 displays the micro-amplification ratio $\mu_{i} / \mu_{j}$ for the main emission regions computed using the results of Table 4 (we have not reported the results for $\mathrm{H} \alpha$ because they are similar to those obtained for $\mathrm{H} \beta$ ). A first quick look at Table 6 shows that, except for the NELs (namely [O II], [Ne V]), the $\mu_{i} / \mu_{j}$ ratio is not compatible with 1 for any value of $(i, j)$. This confirms what we had already revealed in Sect. 3, namely that micro-lensing is occurring in more than one image.

We now examine whether the results are compatible with microlensing occurring in two images. A priori, there are three possible scenarios, depending on whether the un-affected image is assumed to be A, B or C. Thus, each scenario corresponds to $\mu_{\mathrm{A}}=1, \mu_{\mathrm{B}}=1$ or $\mu_{\mathrm{C}}=1$, respectively. From Table 6 it is then easy to derive the respective micro-amplification values $\left(\mu_{\mathrm{B}}, \mu_{\mathrm{C}}\right)$, $\left(\mu_{\mathrm{A}}, \mu_{\mathrm{C}}\right)$ and $\left(\mu_{\mathrm{A}}, \mu_{\mathrm{B}}\right)$, both in the continuum and in the broad components $\mathrm{BC}$ of $\mathrm{H} \beta$ (see values in bold face in Table 6). A robust rule to reject a scenario is the following: the BLR being spatially larger than the continuum emitting region, it cannot be more affected by microlensing than the latter. In other words, the micro-amplification value must be closer to 1 in the BLR for the two affected images in the considered scenario. It is straightforward to check that the above rule is satisfied only in the case when image $B$ is not affected. We find in that case that $\left(\mu_{\mathrm{A}}, \mu_{\mathrm{C}}\right)=$ $(0.28,0.23)$ in the continuum and $\left(\mu_{\mathrm{A}}, \mu_{\mathrm{C}}\right)=(0.80,0.31)$ in $\mathrm{H} \beta$, which means a de-amplification of both images A and $\mathrm{C}$. Both the continuum emitting region and the BLR are affected. We now discuss these results.

${ }^{4}$ The flux ratios measured in [O III] $\lambda \lambda 4959,5007\left(F_{\mathrm{A}} / F_{\mathrm{B}}=1.97\right.$, $\left.F_{\mathrm{C}} / F_{\mathrm{B}}=1.33\right)$ slightly differ from those obtained with the SIE $+\gamma$ fiducial model used in Paper $\mathrm{I}\left(F_{\mathrm{A}} / F_{\mathrm{B}}=1.65, F_{\mathrm{C}} / F_{\mathrm{B}}=0.9\right)$ indicating some inadequation between the model and the data (see Sect. 8.1).

\subsection{Micro-lensing of the BLR}

As already stated in the introduction (Sect. 1), microlensing of the BLR is not surprising in the case of J1131. Here we demonstrate that microlensing has indeed the correct scale to affect the BLR. The Einstein radius $R_{\mathrm{E}}$ of a star of mass $M$ is given by:

$R_{E}=\sqrt{\frac{4 G M}{c^{2}} \frac{D_{\mathrm{ls}} D_{\mathrm{os}}}{D_{\mathrm{ol}}}}=14.3 \sqrt{\frac{M h^{-1}}{M_{\odot}}} \mathrm{lt}-$ days,

where $D_{\mathrm{os}}, D_{\mathrm{ls}}, D_{\mathrm{ol}}$ are the angular-size distances between observer and source (os), lens and source (ls) and observer and lens (ol).

On the other hand, Kaspi et al. (2005) have deduced a relation between the size of the BLR and the luminosity $L_{\lambda}(5100 \AA)$ of the QSO. An improved relation correcting from the host galaxy contamination has been derived in Bentz et al. (2006). Using the magnitude of image B in the $F 814 W$ filter measured with HST (Paper II), and assuming an amplification factor of 10 for that image, we can estimate the likely size of the BLR using Bentz et al.'s relation. We find that the luminosity $\lambda L_{\lambda}(4900 \AA)=$ $7.91 \times 10^{43} h^{-2} \mathrm{erg} / \mathrm{s}$ which translates into a likely size for the $\mathrm{H} \beta$ emission line region of $\sim 40-50$ lt-days (using $h=0.7$; 20-40 lt-days using Kaspi et al.'s relation). Although this can only be a rough estimate due to the bias on the luminosity introduced by the presence of [O III] $+\mathrm{H} \beta$ emission in the $F 814 W$ filter and due to the uncertainty on the macro-lens amplification, we see that a few solar mass micro-lens has an Einstein radius large enough to affect a significant fraction of the broad emission line region.

\subsection{Micro-de-amplification of image $A$}

The micro-de-amplification we find for image A is an independent confirmation of the results found on the basis of flux ratio temporal variability in the optical (Paper I). They also fully support X-ray observations. Indeed, because there is evidence that the QSO X-ray emitting region is smaller than the continuum emitting region (e.g. Pooley 2006), the de-amplification is expected to be stronger in the X-ray than in the optical, at a given epoch. This was clearly the case in April 2004, with $\mu_{A, X}=0.09$ and $\mu_{A, R}=0.46$, as reported in Table 6 from data in Pooley et al. (2006) and in Paper I, respectively. Finally, the parity of image A being negative (saddle point image), de-amplification can easily be produced by substructure(s) of any mass (e.g. Keeton 2003). Given the time variability detected in Paper I, the microlensing regime is favoured rather than the milli-lensing one.

\subsection{Micro-de-amplification of image $C$}

Like for image $\mathrm{A}$, the de-amplification of image $\mathrm{C}$ is possibly supported by the even stronger de-amplification observed in the $\mathrm{X}$-ray domain in April 2004 (i.e. $\mu_{\mathrm{C}, \mathrm{X}}=0.20$ and $\mu_{\mathrm{C}, \mathrm{R}}=0.27$, see Table 6). Note however that while the X-ray data alone cannot discriminate between the micro-de-amplification of $\mathrm{C}$ and the micro-amplification of $\mathrm{B}$, our data only support the former scenario. A sharp drop in the flux of image $\mathrm{C}$ has also been observed between 2004 and 2006 in the X-ray domain (Kochanek et al. 2006). Of course, this is not evidence for de-amplification in 2003 but simply another sign that $\mathrm{C}$ can undergo variable microlensing.

Unlike the parity of image $\mathrm{A}$, the parity of image $\mathrm{C}$ is positive (minimum image). Consequently, it can only be amplified by an 
isolated substructure. This is not only the case with an isothermal clump (Keeton 2003) but also with a point-mass perturber in the scenario of micro-lensing (i.e. in the Chang-Refsdal 1984 lens model). This is so because for a minimum image and $\kappa<1$, we always have $\kappa+\gamma<1$, where $\kappa$ and $\gamma$ are the macro model dimensionless surface mass density and shear values at the position of the considered image. Adding a second point mass perturber does not change the result (Grieger et al. 1989). However, if a non-negligible fraction of the surface mass density of the lens is made of micro-deflectors, the probability to de-amplify a minimum macro-image increases a lot, as illustrated in Fig. 3 of Schechter and Wambsganss (2002). Their M10 model (macroamplification of 10 of a minimum image) represents a situation quite similar to the one of the $\mathrm{C}$ image of $\mathrm{J} 1131$. The observed de-amplification of image $\mathrm{C}$ by a factor $\sim 4$ would constrain the fractional contribution of micro-lenses to be larger than $30 \%$ of the total surface mass density of the lens around image $\mathrm{C}$.

\subsection{Conclusion}

We have shown that the most simple micro-lensing scenario consistent with the published data on J1131 implies that images A and $\mathrm{C}$ are both de-amplified by a micro-lens. The latter also affects the BELs. We have presented supporting arguments of this scenario.

\section{Microlensing as a probe of the QSO structure}

In the previous section, we presented a scenario accounting for the discordant flux ratios measured in the continuum, the BELs and the NELs. We have shown that the data are compatible with microlensing de-amplification of images A and C. Interestingly, both our exploratory and multi-component analyses (Sects. 3 and 4.2) show that the BEL is nearly completely micro-lensed in image $\mathrm{C}$ and partially micro-lensed in image A. This difference of behaviour is likely due to the larger Einstein radius $R_{\mathrm{E}}$ of the micro-lens affecting $\mathrm{C}$. Indeed, the micro-lensed fraction of a given emitting region increases with the $R_{\mathrm{E}}$ of the micro-lens (Eq. (9)). In this context, an emission region that is micro-lensed in image A should also be micro-lensed in image $\mathrm{C}$ and should be very compact, while a larger emitting region might be microlensed only in image C. Finally, even larger regions should not be micro-lensed either in A or in C. Consequently, we are able to infer information about the BEL structure by simply identifying and characterizing the emitting regions microlensed in $\mathrm{A}$ and $\mathrm{C}$.

This scenario further supports our assumption that there is no chromatic micro-lensing of the optical continuum. Indeed, chromatic micro-lensing is expected to be stronger for smaller Einstein radii as well as during caustic crossing events (Wambsganss \& Paczyński 1991). Due to the smaller $R_{\mathrm{E}}$ in image $\mathrm{A}$ than in $\mathrm{C}$ and to the absence of time flux variations of the $\mathrm{B} / \mathrm{C}$ ratio between 2002 and 2004 (Paper I) - indicating that there was no caustic crossing event for image $\mathrm{C}-$, we would expect chromatic micro-lensing of the optical continuum to be stronger in image $\mathrm{A}$ than in $\mathrm{C}$. However, the absence of a chromatic trend in the A/B spectral ratio (Fig. 4) suggests that this effect is negligible. It is thus meaningful to phenomenologically probe the BLR structure using the MCD analysis of A-B-C (Sects. 4.2) and the results of the $F \mu$ decomposition (Sect. 3.2). The adopted micro-lensing scenario provides us with a simpler interpretation of the $F \mu$ decomposition of the A-B and B-C pairs of spectra. Indeed, the absence of micro-lensing in image $\mathrm{B}$ means that the $F \mu$ decomposition of resp. the A-B and B-C pairs unveils the micro-lensed regions in resp. A and $\mathrm{C}$.
We discuss in the next two sections the structural information inferred from the microlensing of the emission line regions (Sect. 6.1) and of the pseudo-continuum emitting region (Sect. 6.2).

\subsection{Microlensing of the emission line regions}

A first interesting result relates to the differences existing between the Mg II and Balmer emission lines. Indeed, the assumed decomposition of the Balmer lines with 2 broad components (BC1 with $F W H M \sim 2000 \mathrm{~km} \mathrm{~s}^{-1}$ and BC2 with $F W H M \sim$ $4300 \mathrm{~km} \mathrm{~s}^{-1}$ ) did not enable us to reproduce the $\mathrm{Mg}$ II profile. The foot of that line is clearly larger than the one of the Balmer lines and is well modelled with a very broad component VBC $\left(F W H M \sim 22000 \mathrm{~km} \mathrm{~s}^{-1}\right)$. Additionally, we found with the MCD method that VBC is significantly de-amplified in both A and $\mathrm{C}\left(\mu_{\mathrm{A}}(\mathrm{VBC})=0.57 ; \mu_{\mathrm{C}}(\mathrm{VBC})=0.25\right.$; Table 6$)$. This is confirmed by the finding of a very broad component in $F_{M \mu}(\mathrm{A})$ and $F_{M \mu}(\mathrm{C})$ at the wavelength of $\mathrm{Mg}$ II (Figs. 5 and 7). Because it is microlensed in both $\mathrm{A}$ and $\mathrm{C}$, the very broad component $\mathrm{VBC}$ in $\mathrm{Mg}$ II should be emitted in a very compact region.

We also found systematic evidence that the 2 broad components $\mathrm{BC} 1$ and $\mathrm{BC} 2$ used to decompose the BELs are differently micro-lensed in image $\mathrm{C}$. We measured $\mu_{\mathrm{C}}(\mathrm{BC} 2)<$ $\mu_{\mathrm{C}}(\mathrm{BC} 1)<1$ for $\mathrm{Mg}$ II, $\mathrm{H} \beta$ and $\mathrm{H} \alpha$. This demonstrates that the broadest component $\mathrm{BC} 2$ is more strongly de-amplified than the narrowest broad-line $\mathrm{BC} 1$, indicating that $\mathrm{BC} 2$ is more compact than $\mathrm{BC} 1$. Additionally, we found with $\mathrm{MCD}$ that $\mu_{\mathrm{C}}(\mathrm{NC} 1)=1$ for the narrow $\mathrm{H} \beta$ emission. Consistently, this narrow component at $z=0.654$ is seen in $F_{M}(\mathrm{C})$ for both $\mathrm{H} \beta$ and $\mathrm{H} \gamma$. However, the $\mathrm{H} \beta$ and $\mathrm{H} \gamma$ profiles in $F_{M}(\mathrm{C})$ cannot be fitted by a single narrow Gaussian profile at $z=0.654$. Especially, the foot of the line is too large, likely due to the differential micro-lensing of the broad component of the line. Contrary to what is observed for the Balmer lines, there is no narrow (not micro-lensed) Mg II emission at $z=0.654$, but a narrow absorption doublet (Sect. 7.2). On the other hand, a symmetric Mg II emission line is observed at the systemic redshift in $F_{M}(\mathrm{C})$. A Gaussian fit of this profile shows that its $F W H M$ is $\sim 2500 \mathrm{~km} \mathrm{~s}^{-1}$. However, we cannot assess whether this component is kinematically isolated (i.e. "narrow" Mg II emission doublet with FWHM $\sim 1000 \mathrm{~km} \mathrm{~s}^{-1}$ ) or not (i.e. associated with differential microlensing of the broad Mg II line).

A more speculative result concerns the microlensing of the Balmer lines in image A. We observe with the $F \mu$ decomposition that only the wings of the $\mathrm{H} \beta$ and $\mathrm{H} \gamma$ profile are affected by some micro-lensing (Fig. 5). On the other hand, the MCD analysis reveals that $\mu_{\mathrm{A}}(\mathrm{BC} 1)>1$ while $\mu_{\mathrm{A}}(\mathrm{BC} 2)<1$. This apparent difference of micro-lensing regime between $\mathrm{BC} 1$ and $\mathrm{BC} 2$ (i.e. amplification of $\mathrm{BC} 1$ and de-amplification of BC2) is likely not a real effect but is due to the MCD method which assumes that Gaussian profiles describing $\mathrm{BC} 1$ and $\mathrm{BC} 2$ are microlensed as a whole ${ }^{5}$. Such a measurement is indeed compatible with the central part of the line profile being less micro-lensed than the wings, such that an excess of flux is measured in the core of the line (i.e. BC1), mimicking a micro-amplification of the latter. Although only the wings of $\mathrm{H} \gamma$ are observed in $F_{M \mu}(\mathrm{A})$, the profile is not an exact replica of $\mathrm{H} \beta$. Especially, the reddest fraction of $\mathrm{H} \gamma$ is narrower than its homologue in $\mathrm{H} \beta$. Additionally, it coincides with possible [O III] $\lambda 4363$ emission at $z=0.654$.

\footnotetext{
5 This assumption is not valid for image A as suggested a priori by the evidence for a partial micro-lensing of the BELs in that image while micro-lensing de-amplify nearly completely the BELs in C.
} 


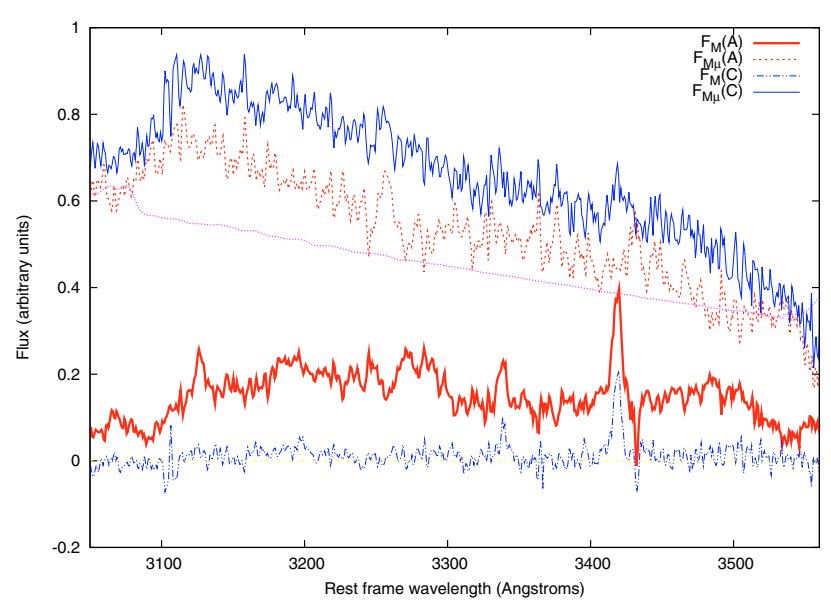

Fig. 9. Zoom on the $3080<\lambda<3540 \AA$ range (rest frame) for $F_{M \mu}(\mathrm{A})$ (dashed red) and $F_{M \mu}(\mathrm{C})$ (thin solid blue) and $F_{M}(\mathrm{~A})$ (thick solid red) and $F_{M}(\mathrm{C})$ (dashed-dotted blue). The pseudo continuum model of $F_{M \mu}$ is overplotted in short dashed pink lines. The emission in $F_{M}(\mathrm{~A})$ is not compatible with 0 and is likely Fe II emission. However, there is a conspicuous excess of flux in $F_{M \mu}(\mathrm{A})$ w.r.t. the model (dotted pink line) which might also be due to Fe II. For image $\mathrm{C}$, all the emission in that range is micro-lensed.

This would reveal that at least a fraction ${ }^{6}$ of [O III $] \lambda 4363$ is coming from a region more compact than the other narrow emission lines (e.g. $\mathrm{H} \beta$, [O III] $\lambda \lambda 4959,5007,[\mathrm{Ne} \mathrm{V}])$ and even as compact as the inner part of the BLR. All these results underline that differential micro-lensing of the BEL is important in image A but cannot be fully understood with our phenomenological approach. Nevertheless, we interestingly notice that micro-lensing of only the wings of the line profile is predicted in Abajas et al. (2002) for an outflowing broad emission region. On the other hand, the existence of a compact [O III] $\lambda 4363$ emission is supported by the work of Nagao et al. (2001a) who show that a significant fraction of [O III] $\lambda 4363$ is likely formed closer to the continuum than the other main NELs. Because the critical density of $[\mathrm{Ne} \mathrm{V}]$ is only two times smaller than for [O III] $\lambda 4363$, one would expect [ $\mathrm{NeV}$ ] to be formed in a region similar to [O III] $\lambda 4363$ (Nagao et al. 2001b) and thus be partially microlensed, which is not observed.

In summary, we have brought several pieces of evidence that the Balmer and Mg II BLRs are different. Especially, we have found that there is a very broad compact component in $\mathrm{Mg}$ II, not present in the Balmer lines. The narrow emission found in $\mathrm{H} \beta$ and $\mathrm{H} \gamma$ at the same redshift as the other NELs (i.e. [O III] $\lambda \lambda 4959,5007,[\mathrm{Ne} \mathrm{V}])$ is absent for Mg II where instead narrow absorption lines are observed. The micro-lensing of the BELs also confirms that the size of the emission region is anticorrelated with the $F W H M$ of the line. Finally, it seems that the micro-lensing of the BELs in image A resolves the BLR in velocity. However, in order to discriminate between different BLR emission models, multi-epoch data as well as a realistic modelling of the BLR (e.g. Abajas et al. 2002; Lewis \& Ibata 2004) are necessary. This will be investigated in a future paper.

\subsection{Micro-lensing of the pseudo-continuum}

The $F \mu$ decomposition of the spectra (Figs. 5 and 7) shows that a significant fraction of the Fe II is microlensed in image $\mathrm{C}$ but

\footnotetext{
6 Because narrow emission -possibly associated with $\mathrm{H} \gamma$ - is also present at this wavelength in $F_{M}(\mathrm{~A})$ and $F_{M}(\mathrm{C})$, it might be that only a fraction of [O III] $\lambda 4363$ is micro-lensed.
}

not in image A. This is confirmed by the MCD analysis for which we measure similar flux ratios in the $\mathrm{Fe} \mathrm{II}_{\mathrm{opt}}$ (and possibly Fe $\mathrm{II}_{\mathrm{UV}}$ ) and in the BELs. This indicates that Fe II is emitted in a region similar to the BLR. This is not surprising since Fe II emission lines are generally broadened like the broadest part of Balmer lines (e.g. Sulentic et al. 2000). Although our result does not prohibit that Fe II emission arises in an accretion disk as generally believed (e.g. Collin-Souffrin et al. 1980; Zhang et al. 2006a), it does not favour a scenario where all the Fe II is emitted in the very inner part of the disk. Nevertheless, we will show hereafter that we have a hint that a fraction of the Fe II emission is emitted in a more compact region.

We can see in $F_{M \mu}(\mathrm{A})$ that there is an excess of flux just blueward of the $\mathrm{H} \beta$ emission (i.e. rest frame range 4630-4800 $\AA$ ). Additionally, emission is still observed at the same wavelength in $F_{M}(\mathrm{~A})$. When $F_{M}(\mathrm{~A})$ (redward of $\mathrm{H} \gamma$ ) is fitted with the Fe II $_{\text {opt }}$ template, we find a lack of emission w.r.t. the template in the range 4630-4800 $\AA$. This suggests that the excess of flux in $F_{M \mu}(\mathrm{A})$ corresponds to Fe II emission missing in $F_{M}(\mathrm{~A})$. In the range $4630-4800 \AA$, Fe II is mainly produced by the four following multiplets: [Fe II] 4F, [Fe II] 20F, Fe II 50 and Fe II 43 (Véron-Cetty et al. 2004). If the micro-lensed Fe II is associated with a particular Fe II transition, one would expect to observe other components of the multiplet associated with that transition elsewhere in $F_{M \mu}(\mathrm{A})$. Among the 4 mentioned multiplets, Fe II 50 and Fe II 43 only emit in the range 4630-4800 $\AA$, but [Fe II] $4 \mathrm{~F}$ is also emitting at $\lambda 4889 \AA$ and [Fe II] 20F is also emitting at $\lambda \lambda 4874,4905 \AA$ and around [O III] $\lambda \lambda 4959,5007$. Since these other components of the multiplet are significantly blended with the $[\mathrm{O} \mathrm{III}]$ and $\mathrm{H} \beta$ emissions, we indeed cannot identify which are the micro-lensed Fe II transition(s). In conclusion, the present data indicate that at least a fraction of Fe II in the range $4630-4800 \AA$ is emitted in a compact region, possibly as compact as the VBC of Mg II.

Similarly to what is observed in the range 4630-4800 , an excess of flux is also observed in the rest-frame range 3080-3540 $\AA$ (where no Fe II templates exist). Indeed, we can see in Fig. 9 that a fraction of the emission in that range is microlensed in A \& $\mathrm{C}$ while another fraction is micro-lensed only in image $\mathrm{C}$. Although it is known that the emission in that range is mainly due to Fe II, we cannot demonstrate that the microlensing behaviour we observe is associated with two different Fe II emitting regions (as for the range 4630-4800 $\AA$ ) or not. If not associated with Fe II, we have no credible emission candidate $^{7}$ for the fraction of the emission (in the range $3080-3540 \AA$ ) micro-lensed in A and C. This open question needs careful identification of the Fe II emission - and absorption - lines in the range $3080-3540 \AA$. This is beyond the scope of the present paper.

\section{Additional results}

\subsection{The NEL extension}

The spectrum extraction of $\mathrm{A}, \mathrm{B}$ and $\mathrm{C}$ has revealed that the spatial profile of the $[\mathrm{O}$ III $] \lambda \lambda 4959,5007$ region is significantly different from the one of the underlying continuum especially for images A and C. Indeed, after extraction, significant residuals

\footnotetext{
7 Due to the steep flux increase observed in the range $~ 3040-3120 \AA$, the 3080-3540 A range was imperfectly modelled with our Balmer continuum model, suggesting that the microlensed flux in $F_{M \mu}(\mathrm{A})$ is not related to the Balmer continuum.
} 


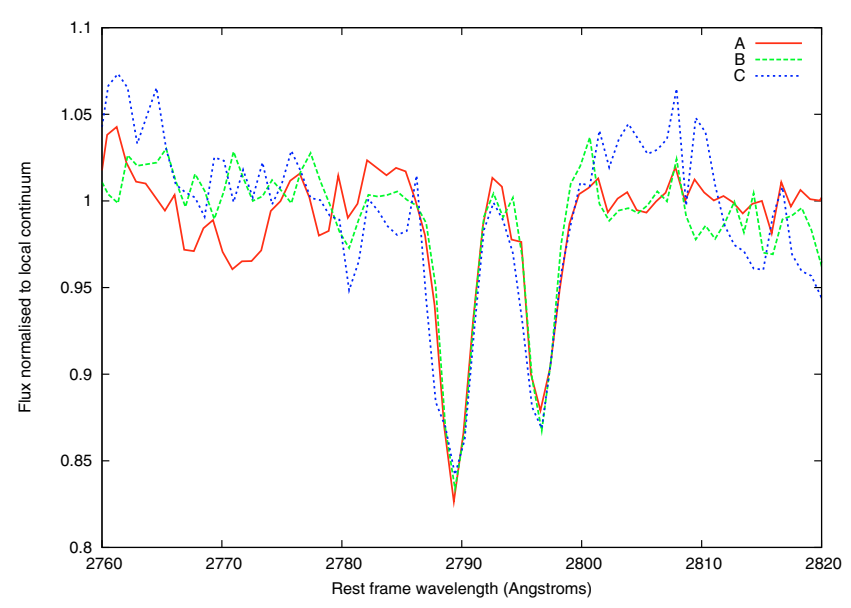

Fig. 10. Associated Mg II absorption doublet normalized to a local effective continuum (i.e. continuum including the broad $\mathrm{Mg}$ II emission) for the 3 lensed images A, B and C. The similar depth in the three images indicates that the origin of the absorption covers both the continuum and the BLR.

are present around both images $\mathrm{A}$ and $\mathrm{C}$ while nothing comparable is observed around image B (Sect. 2.3). This indicates that the flux in the NEL is partly resolved along the slit direction. On the other hand, we did not find a significant offset (i.e. offset $<1$ pixel) of the centroids of the NEL images w.r.t. to the centroid of the continuum emission. Using a Singular Isothermal Ellipsoid + external shear $(\mathrm{SIE}+\gamma)$ to model the lensing galaxy (Paper I), we calculate that the absence of such an offset implies an upper limit of roughly $\sim 30 h^{-1}$ pc on the off-centering of the [O III] emission w.r.t. the continuum emission. Once we have noticed the absence of an off-centering of the [O III] $\lambda \lambda 4959$, 5007 emission, we can safely impose a limit on its size. Indeed, using the SIE $+\gamma$ lens model, we find that images A, B and C are merging for a source size radius ${ }^{8} \geq 110 h^{-1} \mathrm{pc}$. This size is compatible with the typical size of the NLR $(\sim 1 \mathrm{kpc}$; Bennert et al. 2002). Stronger constraints could be derived by combining integral field spectroscopy of J1131 with a proper method to estimate uncertainties due to the seeing, sampling rate and lens model degeneracies (Yonehara 2006).

\subsection{The intrinsic absorption lines}

Another remarkable result is the presence in our spectra of a $\mathrm{Mg}$ II absorption doublet blueshifted at $z=0.654$. According to its velocity shift $\left(\Delta v \sim-660 \mathrm{~km} \mathrm{~s}^{-1}\right)$, this absorption system can be classified as an "associated absorption line" (AALs; see e.g. Hamann \& Sabra 2003).

These lines disappear from the spectrum ratios $\mathrm{A} / \mathrm{B}$ and $\mathrm{C} / \mathrm{B}$. This indicates that the absorbed flux is proportional to the flux coming from the continuum+BLR. This implies that the region at the origin of the absorption must cover both the continuum and the BLRs and that, within the uncertainties, their depths are identical in the spectra of the three images A, B, and C. This is clearly illustrated in Fig. 10 where the absorption lines are normalized to a local effective "continuum" which includes the broad Mg II emission line.

The relative intensities of the blue and red lines of the doublet show evidence for partial coverage. Indeed if $I_{\text {blue }}$ and $I_{\text {red }}$

\footnotetext{
${ }^{8}$ We should notice that this estimate is model dependent. If e.g. multipoles are added to the lens model (Paper II), one finds that images A, $\mathrm{B}$ and $\mathrm{C}$ are merging for emission regions $>36 h^{-1} \mathrm{pc}$.
}

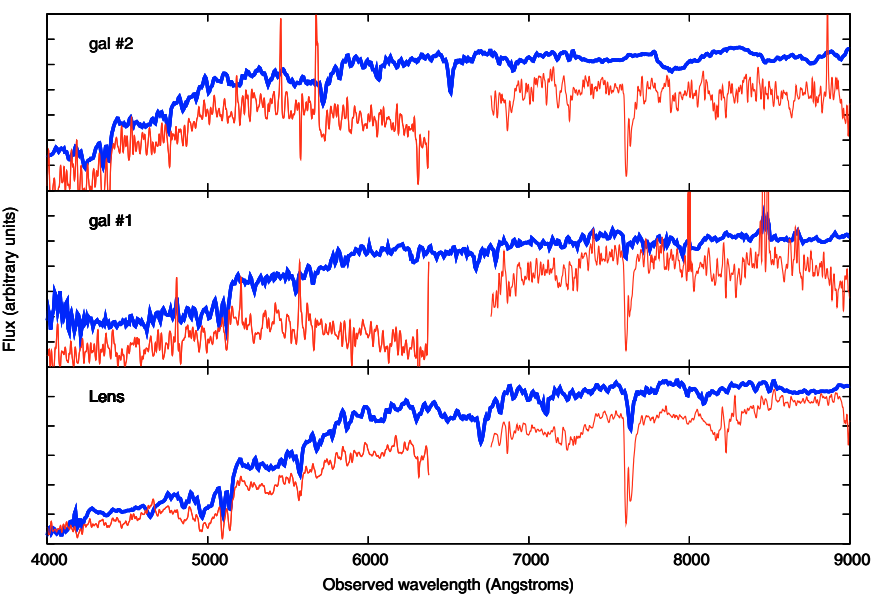

Fig. 11. Smoothed spectra in the blue and red ranges (thin solid red) of the lensing galaxy (bottom panel), gal \#1 (middle panel) and gal \#2 (top panel) and associated redshifted template spectra (thick solid blue). A flux offset has been imposed for legibility. Due to mis-centering of gal $\# 1$ and \#2 in the slit, there is significant slit losses for $5500<\lambda<$ $6500 \AA$ and $\lambda>8500 \AA$. Best redshifts are found to be $z=0.295$ (Lens), $z=0.289(\mathrm{gal} \mathrm{\# 1})$ and $z=0.105$ (gal \#2).

are the normalized residual intensities of the two lines of the doublet and $\tau$ the optical depth, we expect $I_{\text {blue }}=\mathrm{e}^{-\tau}$ and $I_{\text {red }}=\mathrm{e}^{-0.5 \tau}$ in the case of full uniform coverage. The fact that $I_{\text {red }}^{2}<I_{\text {blue }}$ as clearly seen in Fig. 10 suggests that only a part of the local continuum is covered by the absorption line region. Assuming homogeneous partial coverage (Hamann \& Sabra 2003), we find from these intensities that the covering factor of the absorption region is around $20 \%$ (see e.g. the relations given in Hutsemékers et al. 2004). This suggests that the absorption is intrinsic to the quasar, and exterior to the BLR.

One can see the differential microlensing at work in J1131 as a probe of the inhomogeneities in the absorbing medium. Indeed, by lensing more (less) strongly some regions of the source, differential micro-lensing increases (decreases) the contribution of a fraction of the intervening absorber to the total absorption. The nearly identical absorption depths seen in the three images (Fig. 10) indicates that both the spatial distribution and the optical depth of the absorbing clouds must be quite homogeneous over the continuum and BLRs. This is compatible with an absorption region composed of a large number of small absorbing clouds, their projected sizes being significantly smaller than the continuum region. A more quantitative study of this effect needs modelling of the absorbing medium, of the micro-lensing event as well as higher spectral resolution and $S / N$ data.

\subsection{The lensing galaxy and neighbours}

The slit orientation \#2 provides us with a medium resolution spectrum of the lensing galaxy and of two neighbour galaxies gal \#1 and \#2 located at 55" and 95" from the lens (Sect. 2.3). We confirm the identification of the lensing galaxy by Sluse et al. (2003) as an elliptical galaxy at $z=0.295$. Unfortunately, the $\mathrm{S} / \mathrm{N}$ is too poor to derive the central velocity dispersion. The galaxy gal\# 1 is identified on deep $R$-band imaging as a face-on spiral galaxy. There are 2 strong emission features with an intensity ratio $2: 1$ observed at $\lambda \lambda 8461.5$ \& $8488 \AA$ and a fainter one at $\lambda 8440 \AA$. We identify these lines as $\mathrm{H} \alpha \lambda 6562$ and $[\mathrm{N} \mathrm{II}] \lambda \lambda 6548,6583$ emission associated with intense star formation. A careful look at the smoothed 
spectrum of the galaxy reveals emission of [S II] $\lambda \lambda 6716,6731$, [O II] $\lambda 3727$ and $\mathrm{H} \gamma \lambda 4340$ that definitely confirm a redshift value of $z=0.289 \pm 0.002$ for this galaxy. Once smoothed with a Gaussian kernel, the spectrum of the second galaxy (gal\#2) clearly reveals several absorption features among which $\mathrm{Ca} \mathrm{K}$ and $\mathrm{H} \lambda \lambda 3933.7,3968.5 \AA$. The redshift of gal \#2 derived from these absorption lines is $z=0.105 \pm 0.002$. The spectra of these galaxies compared to the associated redshifted templates are displayed in Fig. 11.

Gal\#1 and gal\#2 are in the direction of the external shear (with the convention that the shear $\gamma$ points towards or opposite to the perturber). If we model the perturbing galaxy as a Singular Isothermal Sphere, we can calculate the amount of shear caused by that perturber (e.g. formula A.20. of Momcheva et al. 2006). Using a conservatively large value $\sigma=300 \mathrm{~km} \mathrm{~s}^{-1}$ for the velocity dispersion, we estimate that the amount of shear caused by gal\#1 and gal\#2 is only $\gamma \sim 0.011$ for each galaxy9. Even then, this is not sufficient to explain the shear value $\gamma \sim 0.1$ necessary in the lens models (e.g. Papers I and II). This suggests that these two perturbers might be members of more massive galaxy groups. Interestingly, Williams et al. (2006) - based on two band imaging and red sequence finding technique - found evidence for two galaxy groups likely at $z=0.19$ and $z=0.29$ in the field of J1131. The redshift of gal\#2 together with the measured redshift $z=0.1035$ and $z=0.1006$ of two other field galaxies obtained during the Las Campanas redshift survey (Shectman et al. 1996) suggest a redshift $z \sim 0.1$ for the foreground galaxy group. These two possible groups might play a role in the modelling of that system. This will be further investigated elsewhere.

\section{Caveats}

In this section, we first further discuss the use of [O III] as a reasonable estimate of the macro-lens model flux ratios. Second we estimate whether the results might be affected by the time delay between A-B-C. Third, we briefly address the question of the effect of intrinsic quasar absorber that might modify the BEL profile independently of any micro-lensing.

\subsection{Macro-amplification ratios}

Although it seems reasonable to use the flux ratios in [O III] as an estimate of the macro-model flux ratio, one may ask how robust our conclusions are with respect to that assumption. For this purpose, we can compare the flux ratios in [O III] to those expected by a simple smooth lens model. Using the SIE $+\gamma$ model derived in Paper I as a fiducial model, we find $F_{\mathrm{A}} / F_{\mathrm{B}}=1.65$ and $F_{\mathrm{C}} / F_{\mathrm{B}}=0.9$ while the values measured in [O III] are $F_{\mathrm{A}} / F_{\mathrm{B}}=1.97$ and $F_{\mathrm{C}} / F_{\mathrm{B}}=1.33$. Although there are significant differences likely due to an imperfect modelling of the lens potential, the values measured in [O III] are more similar to the model than those measured in the BEL or in the continuum. Therefore, using the fiducial model estimates of the macro model flux ratios will not change our conclusions. Indeed, using the macro model values instead of the [O III] flux ratios as a proxy of the macro-amplification ratios would only change the amplitude of micro-lensing in $\mathrm{A}$ and $\mathrm{C}$ but nothing else.

One may ask whether the [O III] emitting region is large enough to be unaffected by micro-lensing. Indeed, using Eq. (9), one find that a region larger than $100 \mathrm{pc}$ can be significantly microlensed by substructures more massive than typically

9 Although the angular separation from the lens is larger for gal\#2, its shear amplitude is as high as for gal\#1 because of its smaller redshift.
$5 \times 10^{7} M_{\odot}$. Such a massive substructure is unlikely to affect image A. Indeed, if present, it should significantly modify the BEL while we have evidence that the latter is only marginally deamplified compared to the continuum. On the other hand, millilensing of image $\mathrm{C}$ is more likely since we observe a significant de-amplification of a large fraction of the BLR. Consequently, we may hypothesize that the NLR in C is partly demagnified. The only consequence of such an assumption would be that the effect of micro-lensing of the continuum and the BELs in $\mathrm{C}$ are stronger than estimated. Alternatively, magnification (instead of de-magnification) of the NLR seems incompatible with the data. Indeed, if the NLR is magnified by substructures, one expects that the BLR and the continuum will be more strongly affected, which is not observed. Thus, only an ad-hoc scenario in which the NLR is magnified by a massive substructure (e.g. a dwarf galaxy) and the BELs and continuum are de-magnified by stars (e.g. the stars in the dwarf) would be compatible with the data.

\subsection{Bias due to the time delay}

Recently, Morgan et al. (2006) argued that the time-delay between $\mathrm{A}$ and $\mathrm{B}(\mathrm{C})$ is around 15 days. One may thus imagine that time variability between A and B (C) may play a role in the interpretation of our measurements. However, over the nearly 2 years of monitoring performed by Morgan et al., intrinsic variability never exceeded 0.05 mag over a period of 15 days. Intrinsic variability may thus be considered as an additional source of noise affecting our measurements at a level of typically $5 \%$. This is not only true for the continuum but also for the BEL that is mainly formed through photoionisation processes which imply that the BEL flux variations respond linearly to the flux variations of the continuum (e.g. Peterson et al. 1985). Consequently, the timedelay between A and B (C) should only marginally affect our results.

\subsection{Bias due to intrinsic absorbers}

Green (2006) recently investigated how the small line of sight differences existing between lensed images may modify the observed emission line profiles. Green suggests that some warm absorber outflow located close to the QSO continuum might modify the BEL line profile since it would be present along the line of sight of one image but not of the other. However, if intrinsic absorbers modify the BEL in A-B-C it is unrealistic that they would affect the whole emission profile. Partial "deformation" of the broad line profiles due to a warm absorber might bias the $F \mu$ decomposition of the BELs (Sect. 3.2), because the method assumes a similar intrinsic profile in each component. The similarity between the $\mathrm{B}$ and $\mathrm{C}$ line profiles suggests that BEL differences due to line-of-sight effects are not significant in these images. If present, this effect likely occurs in image A for which the $\mathrm{H} \beta$ line profile appears more asymmetric. However, $\mathrm{H} \beta$ absorbers are very rare: up to now, broad absorption lines in $\mathrm{H} \beta$ have been observed only in 4 systems (Hall 2006). Long term spectroscopic monitoring will shed light on such an effect if present at all.

\section{Conclusions}

This paper is devoted to a thorough analysis of the long slit spectra obtained for the gravitationally lensed quasar J1131-1231. The spectra of the three brightest images A-B-C enabled us to estimate the flux ratios in different emitting regions (namely the 
continuum, the broad line and the narrow line emitting regions) and unveil the micro-lensing effects occurring in that system. Evidence for differential micro-lensing of the broad emission lines enabled us to perform a phenomenological study of the structure of the Broad Line emitting Region (BLR).

Due to the better resolving power of the present spectra, new redshift estimates of the source and of the lensing galaxy have been performed. Based on the Mg II emission, a systemic redshift $z=0.657 \pm 0.001$ has been deduced. The narrow emission lines (except [O II] $\lambda 3727 ; z=0.656 \pm 0.001$ ) are blueshifted w.r.t. the systemic redshift. A redshift $z=0.654 \pm 0.001$ is measured for those lines. On the other hand, we confirm the redshift of the lensing galaxy to be $z=0.295 \pm 0.001$. Finally, we have estimated the redshifts of the 2 galaxies gal \#1 and \#2 located at resp. 55" and 95" from the lens to be resp. $0.289 \pm 0.002$ and $0.105 \pm 0.002$.

The results derived from the micro-lensing analysis and its implications for the quasar structure are summarized below:

1. Different flux ratios were derived for the continuum, the Broad Emission Lines (BELs) and the Narrow Emission Lines (NELs), indicating that micro-lensing is at work in at least 2 images. The simplest scenario explaining our observations consists in micro-lensing de-amplification of images $\mathrm{A}$ and $\mathrm{C}$. Additionally, we found evidence for differential micro-lensing of the $\mathrm{BLR}^{10}$. Since a larger fraction of the BELs is micro-lensed in image $\mathrm{C}$, the Einstein radius of the micro-lens is likely larger in $\mathrm{C}$ than in A. Using the relation between the size of the BLR and the luminosity of the QSO (Kaspi et al. 2005; Bentz et al. 2006), we found that a microlens of a few solar masses is sufficient to affect the whole BLR.

2. We found that the MgII and the Balmer emission lines cannot be represented with the same velocity components. Especially, the very broad component of the Mg II emission is not present in the Balmer lines. Since it is micro-lensed in image $\mathrm{C}$ and $\mathrm{A}$, this component likely comes from a very compact region.

3. The differential micro-lensing of the BELs confirms that the size of an emission line region is anti-correlated with the $F W H M$ of the corresponding line component.

4. We have an indication that differential micro-lensing in image $\mathrm{A}$ resolves the BLR in velocity. Monitoring of such an effect combined with modelling of the BLR offers interesting avenues to probe the BLR structure and kinematic.

5. We found that the narrow emissions observed in the Balmer lines have the same characteristics as the [O III] $\lambda \lambda 4959$, 5007 narrow emission, namely same width, redshift and magnification ratio. This argues in favour of a common emitting region. On the other hand, we rule out the existence of a similar narrow emission for $\mathrm{Mg}$ II.

6. An intriguing narrow micro-lensed emission incidentally coincides with the [O III] $\lambda 4363$ emission, suggesting a very compact emission region for that line. The robustness of this result is however questionable. Indeed, this emission might also be produced by the differential micro-lensing of $\mathrm{H} \gamma$. Additionally, one would expect to see a similar behaviour

${ }^{10}$ During the referee process, Sugai et al. (2007) published an analysis of IFS data of J1131 in the $\mathrm{H} \beta-[\mathrm{O}$ III] $\lambda \lambda 4959,5007$ range. Although obtained at a different epoch (Feb. 2005), these data display emission line flux ratios quite similar to those presented here and interpreted as micro-lensing of images A and C. Similarly to our results, these data also suggest differential micro-lensing of the BLR as well as spatially resolved [O III] emission. for e.g. [ $\mathrm{Ne} \mathrm{V}]$ emission, which is not observed. This result clearly needs further investigation.

7. The $[\mathrm{O}$ III $] \lambda \lambda 4959,5007$ narrow emission lines are partly spatially resolved. Neglecting seeing, resolution effects, and lens model degeneracies, we argue that it provides us with a lower limit on the size of the NLR of $110 h^{-1} \mathrm{pc}$, fully consistent with typical NLR size.

8. We have firm evidence that a large fraction of the near-UV and optical Fe II emission arises in the outer parts of the BLR. We have however shown that a smaller fraction of the $\mathrm{Fe}$ II is emitted in the inner parts of BLR. Compact emission from Fe II is very likely identified in the rest frame range 4630-4800 ̊. Micro-lensed pseudo continuum emission is present in the range $3080-3540 \AA$. This emission is likely associated with Fe II.

9. An associated absorption line doublet was observed in $\mathrm{Mg}$ II at the same redshift as the NELs. We have found that the absorbing medium is intrinsic to the QSO and covers both the continuum and BLR emission, with a covering factor of $\sim 20 \%$. Additionally, using differential micro-lensing as a probe of the inhomogeneities in the absorbing medium, we have shown that both the spatial distribution and the optical depth of the absorbing clouds are homogeneous over the BLR and the continuum.

Although our phenomenological description of the microlensing of the BELs is imperfect in several aspects, it nicely illustrates the wealth of information one can retrieve from spectroscopic observations of micro-lensed QSOs. Clearly, more detailed models as well as coordinated spectroscopic (integral field) monitoring of lensed quasars where micro-lensing takes place will improve our understanding of both the micro-lensing phenomenon and of the QSO structure.

Acknowledgements. We thank M. Vestergaard for kindly providing us her UV Fe II template and Monique Joly for useful discussions about Fe II emission in quasars. We also acknowledge Chris Lidman for his tips on the NIR spectral reduction and wavelength calibration. DS is supported by the Swiss National Science Foundation. JFC and JS acknowledge support from the ESA PRODEX Programme "HST Imaging of Field and Gravitationally Lensed Quasars" and the Belgian Federal Science Policy Office for their support.

\section{References}

Abajas, C., Mediavilla, E., Muñoz, J. A., Popović, L. Č., \& Oscoz, A. 2002, ApJ, 576,640

Avila, G., Rupprecht, G., \& Beckers, J. M. 1997, SPIE, 2871, 1135

Bennert, N., Falcke, H., Schulz, H., Wilson, A. S., \& Wills, B. J. 2002, ApJ, 574, L105

Bentz, M. C., Peterson, B. M., Pogge, R. W., Vestergaard, M., \& Onken, C. A. 2006, ApJ, 644, 133

Boroson, T. A., \& Green, R. F. 1992, ApJS, 80, 109

Chang, K., \& Refsdal, S., 1984, A\&A, 132, 168

Claeskens, J.-F., Sluse, D., Riaud, P., \& Surdej, J. 2006, A\&A, 451, 865 (Paper II) Corbett, E. A., Croom, S. M., Boyle, B. J., et al. 2003, MNRAS, 343, 705

Collin-Souffrin, S., Joly, M., Dumont, \& S., Heidmann, N. 1980, A\&A, 83, 190 Dietrich, M., Hamann, F., Appenzeller, I., \& Vestergaard, M. 2003, ApJ, 596, 817

Grandi, S. A. 1982, ApJ, 255, 25

Green, P. J. 2006, ApJ, 644, 733

Grieger, B., Kayser, R., Refsdal, S., \& Stabell, R. 1989, The two point mass gravitational lens in Abhandlungen aus der Hamburger Sternwarte X, 4 Hamburg-Bergedorf

Hall, P. B., [arXiv: astro-ph/0611922]

Hamann, F., \& Sabra, B. 2003, ASPC, 311, 203

Hutsemékers, D., Hall, P. B., \& Brinkmann, J. 2004, A\&A, 415, 77

Jean, C., \& Surdej, J. 1998, A\&A, 339, 729

Kaspi, S., Smith, P. S., Netzer, H., et al. 2000, ApJ 533, 631

Kaspi, S., Maoz, D., \& Netzer, H. 2005, ApJ, 629, 61

Keeton, C. R. 2003, ApJ, 584, 664 
Keeton, C. R., Gaudi B. S., \& Petters A. O. 2003, ApJ, 598, 138

Kinney, A. L., Bohlin, R. C., Calzetti, D., Panagia, N., \& Wyse, R. F. G. 1993, ApJS, 86, 5

Kochanek, C. S., Dai, X., Morgan, C., et al. 2006, [arXiv:astro-ph/0609112]

Laor, A., Jannuzi, B. T., Green, R. F., \& Boroson, T. A. 1997, ApJ, 489, 656

Lewis, G. F., \& Ibata, R. A. 2004, MNRAS, 348, 24

Mc Lure, M. J., \& Jarvis, R. J. 2002, MNRAS, 337, 109

Momcheva, I, Williams, K. A., Keeton, C. R., \& Zabludoff, A. I. 2006, ApJ, 641, 169

Morgan, N. D., Kochanek, C. S., Falco, E. E., \& Dai, X. [arXiv: astro-ph/0605321], v1

Nagao, T., Murayama, T., \& Taniguchi, Y. 2001a, ApJ, 549, 155

Nagao, T., Murayama, T., \& Taniguchi, Y. 2001b, PASJ, 53, 629

Natali, F., Giallongo, E., Cristiani, S., \& La Franca, F. 1998, AJ, 115, 397

Patnaik, A. R., Browne, I. W. A., Walsh, D., Chaffee, F. H., \& Foltz, C. B. 1992, MNRAS, 259,

Peterson, B. M., Crenshaw, D. M., \& Meyers, K. A. 1985, ApJ, 298, 283

Pooley, D., Blackburne, J. A., Rappaport, S., \& Schechter, P. L. 2006, [arXiv:astro-ph/0607655] (v1)

Romano, P., Zwitter, T., Calvani, M., \& Sulentic, J. 1996, MNRAS, 279, 165

Rousselot, P., Lidman, C., Cuby, J.-G., Moreels, G., \& Monnet, G. 2000, A\&A, 354,1134
Schechter P. L., \& Wambsganss, J. 2002, AJ, 580, 685

Shectman, S. A., Landy, S. D., Oemler, A. et al. 1996, ApJ, 470, 172

Sheinis, A. I. 2006, SPIE, 6269, 99 [arXiv: astro-ph/0606176]

Sluse, D., Surdej, J., Claeskens, J.-F., et al. 2003, A\&A, 406, L43

Sluse, D., Claeskens, J.-F., Altieri, B., et al. 2006, A\&A, 449, 539 (Paper I)

Sugai, H. et al. 2007, ApJ, accepted, [arXiv:astro-ph/0702392]

Sulentic, J. W., Marziani, P., \& Dultzin-Hacyan, D. 2000, ARA\&A, 38, 521

Tsuzuki, Y., Kawara, K., Yoshii, Y., et al. 2006, ApJ, 650, 57

Vanden Berk, D. E., Richards, G. T., Bauer, A., et al. 2001, AJ, 122, 549

Véron-Cetty, M.-P., \& Véron, P. 2000, A\&ARv, 10, 81

Véron-Cetty, M.-P., Joly, M., \& Véron, P. 2004, A\&A, 417, 515

Véron-Cetty, M.-P., Joly, M., Véron, P., et al. 2006, A\&A, 451, 851

Vestergaard, M., \& Wilkes, B. J. 2001, ApJS, 134, 1

Vettering, W. T., Teutolsky, S. A., Press, W. H., et al. Obs, 113, 1115, 214

Wambsganss, J., \& Paczyński B. 1991, AJ, 102, 864

Williams, K. A., Momcheva, I., Keeton, C. R., Zabludoff, A. I., \& Lehár, J. 2006, ApJ, 646, 85

Wills, B. J., Netzer H., \& Wills, D. 1985, ApJ, 288, 94

Wise, J. H., Eracleous, M., Charlton, J. C., \& Ganguly, R. 2004 ApJ, 613, 129 Yonehara, A. 2006 ApJ, 646, 16

Zhang, X. G., Dultzin-Hacyan D., \& Wang, T. G. 2006a, MNRAS, 372, L25

Zhang, X. G., Dultzin-Hacyan D., \& Wang, T. G. 2006b, RMxAA

[arXiv:astro-ph/0610432] 